\title{
A Numerical Scheme Based on the Chebyshev Functions to Find Approximate Solutions of the Coupled Nonlinear Sine-Gordon Equations with Fractional Variable Orders
}

\author{
MohammadHossein Derakhshan \\ Industrial Engineering Department, Apadana Institute of Higher Education, Shiraz, Iran \\ Correspondence should be addressed to MohammadHossein Derakhshan; m.h.derakhshan.20@gmail.com
}

Received 12 September 2020; Revised 19 November 2020; Accepted 12 February 2021; Published 9 March 2021

Academic Editor: Simeon Reich

Copyright (c) 2021 MohammadHossein Derakhshan. This is an open access article distributed under the Creative Commons Attribution License, which permits unrestricted use, distribution, and reproduction in any medium, provided the original work is properly cited.

\begin{abstract}
In this article, a numerical method based on the shifted Chebyshev functions for the numerical approximation of the coupled nonlinear variable-order fractional sine-Gordon equations is shown. The variable-order fractional derivative is considered in the sense of Caputo-Prabhakar. To solve the problem, first, we obtain the operational matrix of the Caputo-Prabhakar fractional derivative of shifted Chebyshev polynomials. Then, this matrix and collocation method are used to reduce the solution of the nonlinear coupled variable-order fractional sine-Gordon equations to a system of algebraic equations which is technically simpler for handling. Convergence and error analysis are examined. Finally, some examples are given to test the proposed numerical method to illustrate the accuracy and efficiency of the proposed method.
\end{abstract}

\section{Introduction}

The basic and important applications of differential equations with fractional variable order which are considered as a generalization of ordinary differential equations with integer order in various fields of computational science [1-3], engineering [4-6], physics $[7,8]$, and chemistry $[9,10]$ have attracted significant attention in the literature. One of the important discussions in differential equations is the study of nonlinear partial and nonlinear differential equations that are used in physics and applied mathematics such as the sine-Gordon equation which is a significant nonlinear integrable evolution partial differential system in space-time coordinates [11]. The coupled nonlinear partial differential sine-Gordon equations of integer order on the interval $(p, q) \in[a, b] \times[0, T]$ are defined by [12]

$$
z_{q q}(p, q)-z_{p p}(p, q)+\theta^{2} \sin (z(p, q)-v(p, q))=h_{1}(p, q)
$$

$$
v_{q q}(p, q)-\xi^{2} v_{p p}(p, q)-\sin (z(p, q)-v(p, q))=h_{2}(p, q)
$$

where symbols $\theta$ and $\xi^{2}$ are the ratios of the acoustic velocities of the functions $z, v$, and the ratios of masses of particles in the "lower" and "upper" parts of the crystal given in the generalized Frenkel-Kontorova dislocation equation [12-14] are the defined parameters $\theta$ and $\xi^{2}$, respectively, which are known, and the functions $z(p, q), v(p, q)$ and $h_{1}(p, q), h_{2}(p, q)$ are unknown and known, respectively. If in Equation (2), $\xi=1$ is considered, then Equation (2) explains the open states in deoxyribonucleic acid (DNA) [15], and if in Equation (2), $\xi^{2} \neq 1$ is considered, then Equation (2) shows a gap in the spectrum of velocities of solitary waves [12]. This paper focuses on finding approximate solutions based on the Chebyshev function scheme for fractional differential equations of variable orders which are called coupled nonlinear sine-Gordon equations, and these type of equations of fractional 
variable orders $\alpha_{1}(p, q), \alpha_{2}(p, q) \in(1,2]$ are defined as follows:

$$
{ }^{C-P} \mathbb{D}_{q}^{\alpha_{1}(p, q)} z(p, q)-{ }^{C-P} \mathbb{D}_{q}^{\alpha_{1}(p, q)} z(p, q)+\theta^{2} \sin (z-v)=h_{1}(p, q),
$$

$$
{ }^{C-P} \mathbb{D}_{q}^{\alpha_{2(p, q)}} v(p, q)-\xi^{2 C-P} \mathbb{D}_{q}^{\alpha_{2(p, q)}} v(p, q)-\sin (z-v)=h_{2}(p, q),
$$

where ${ }^{C-P} \mathbb{D}$ is the Caputo-Prabhakar operator. Also, the initial and boundary conditions for problems (3) are considered as follows:

$$
\begin{aligned}
& z(p, 0)=l_{0}(p), z_{q}(p, 0)=l_{1}(p), \\
& v(p, 0)=l_{2}(p), v_{q}(p, 0)=l_{3}(p), \\
& z(a, q)=f_{0}(q), z(b, q)=f_{1}(q), \\
& v(a, q)=f_{2}(q), v(b, q)=f_{3}(q),
\end{aligned}
$$

where functions $l_{0}(p), l_{1}(p), l_{2}(p), l_{3}(p)$ and $f_{0}(q), f_{1}(q), f_{2}$ $(q), f_{3}(q)$ are continuous. The Caputo-Prabhakar operator considered in this article is an extension of the Caputo derivative which is introduced in [16], and it is defined by

$$
\mathbf{D}_{0^{+}}^{\mu_{1}} z(x)=\mathbf{I}_{0^{+}}^{n-\mu_{1}} \frac{d^{n}}{d x^{n}} z(x)
$$

where $n-1<\mu_{1} \leq n, z \in L^{1}[0, b], b \in \mathbb{R}$ and $\mathbf{I}$ is the Riemann-Liouville integral (RLI). So by putting a function containing the Prabhakar function which is studied in [17], in Equation (9), by developing the RLI kernel with its function, this function is given by [17]

$$
e_{\mathrm{Q}, v}^{v}(x)=x^{\nu-1} E_{\mathrm{Q}, v}^{v}\left(\omega x^{\varrho}\right), \mathfrak{R}(\varrho)>0, \mathfrak{R}(v)>0, \quad x \in \mathbb{R}, \varrho, v, v, \omega \in \mathbb{C},
$$

where $E_{\mathrm{Q}, v}^{v}\left(\omega x^{\mathrm{e}}\right)$ is the Prabhakar function and is defined as follows:

$$
E_{\mathrm{\varrho}, v}^{v}\left(\omega x^{\varrho}\right)=\sum_{l=0}^{\infty} \frac{\Gamma(v+l) x^{l} / l ! \Gamma(v)}{\Gamma(\varrho l+v)} .
$$

Some applications of the Prabhakar function can be seen in mathematics and physics as a fractional Poisson process [16], Havriliak-Negami relaxation functions [18, 19], irregular case of the dielectric relaxation responses [20], a model of anomalous relaxation in dielectrics of fractional order [21], fractional thermoelasticity [10], telegraph equations [22], thermodynamics [23], and fractal time random [24]. By placing $\alpha_{1}(p, q)=2, \alpha_{2}(p, q)=2$ in Equation (3), the coupled nonlinear sine-Gordon equations of fractional variable orders given in (3) change into the classical coupled nonlinear sine-Gordon equations which are defined by Equation (2), and the classical coupled nonlinear sine-Gordon equations have many applications in physics as nonlinear models [25, 26], plasma [27], quantum [28], optics [29], and mathematics [13, 30, 31]. Getting analytic solutions to fractional differential equations in general are not easy; therefore, numerical methods are used to obtain the solutions of this type of equations. For instance, [32] defined a new method based on the linearization formula to find the fuzzy approximate solution of fractional differential equations under uncertainty, a new spectral tau method for solving equations of Kelvin-Voigt equations is proposed in [33], and [34] studied a numerical method based on the Bernstein functions to solve the linear cable equation of variable order. An implicit RBF meshless method [35], spectral method [36], homotopy analysis scheme [37], meshless method [38], collocation and finite difference-collocation methods [39], Ritz-Galerkin method [40], new collocation scheme for solving fractional partial differential equations [41], Sinc-Chebyshev collocation scheme [42], numerical method based on the shifted Chebyshev functions [43], numerical method based on the shifted Legendre functions [44], piecewise integroquadratic spline interpolation and finite difference method [45], wavelet scheme [46], Legendre wavelet method [47], Bernoulli wavelet method [48], and Lagrange multiplier scheme [49] and other methods [50-54]. Several methods are presented for solving variable-order fractional equations. For example, Bhrawy and Zaky [55] used the shifted Jacobi polynomials to obtain solution variable-order fractional Schrödinger equations, Bhrawy and Zaky [56] studied the Jacobi-Gauss-Lobatto collocation method to obtain solution variable-order fractional Schrödinger equations, Mahmoud et al. [57] proposed the Jacobi wavelet collocation method to obtain solution variable-order fractional equations, and Zaky et al. [58] proposed the shifted Chebyshev polynomials to obtain solution variable-order fractional equations; in [59], a proper discrete form of fractional Grönwall-type inequality is introduced and other methods such as shifted Jacobi collocation method [60] and shifted Jacobi method [61]. Since this mathematical system which is given in Equation (3), due to the variable-order fractional operators and nonlinearity, is very complex, we need to reduce it by using a highly accurate and efficient expansion scheme. Thus, for this aim, we first extract an operational matrix of variable-order fractional derivatives for the shifted Chebyshev functions, then we use them for extending the unknown solution. In other words, the problem shown in Equation (3) is converted into an algebraic system of equations by exploiting the operational matrix of variable-order derivative. In this paper, we consider a class of coupled nonlinear variable-order fractional sine-Gordon equations. For solving the given equations, operational matrices based on the shifted Chebyshev functions are applied. First, we approximate the unknown function and its derivatives in terms of the shifted 
Chebyshev functions. Then, by substituting these approximations into the equation and applying the properties of the shifted Chebyshev functions together with the collocation points, the main problem is reduced to a set of nonlinear algebraic equations. By solving this system, the approximate solution is calculated. This article is divided into the following sections. In Section 2, we state the essential definitions and lemmas about fractional derivatives and integrals of the variable order, also in this section are introduced the Chebyshev polynomials and the shifted Chebyshev polynomials. The approximate function is obtained in Section 3. In Section 4, an explanation of the suggested method for obtaining approximate solutions of the equations introduced in (3) is provided and four numerical examples are shown in Section 5.

\section{Preliminaries of Fractional Calculus}

This section discusses the important topics of fractional derivative and integral of the variable order such as the Prabhakar integral and the Caputo-Prabhakar derivative. The Chebyshev polynomials and the shifted Chebyshev polynomials are introduced in this section.

Definition 1. The Prabhakar integral of variable order $\mathfrak{R}$ $(\mu(p, q))>0, q \geq 0$ is defined by $[17,62]$

$$
\begin{aligned}
& { }^{1} \varepsilon_{\mathrm{Q}, \mu(p, q), \omega, 0}^{v}+z(p, q)=\int_{0}^{q}(q-\tau)^{\mu(p, q)-1} E_{\mathrm{Q}, \mu(p, q)}^{v}\left(\omega(q-\tau)^{\mathrm{\varrho}}\right) z(p, \tau) d \tau, \\
& { }^{2} \varepsilon_{\mathrm{Q}, \mu(p, q), \omega, 0}^{v}+z(p, q)=\int_{0}^{p}(p-\tau)^{\mu(p, q)-1} E_{\mathrm{Q}, \mu(p, q)}^{v}\left(\omega(p-\tau)^{\mathrm{\varrho}}\right) z(\tau, q) d \tau,
\end{aligned}
$$

where $f \in L^{1}(0, \infty)$. Also, for $v=0$, the Prabhakar integral coincides with the Riemann-Liouville fractional integral of variable order $\mathfrak{R}(\mu(p, q))>0, q \geq 0$.

Definition 2. The Caputo-Prabhakar derivative of variableorder $\alpha_{1}(p, q), n-1<\alpha_{1}(p, q) \leq n, n \in \mathbb{N}, q \geq 0$ is defined by $[17,62,63]$

$$
\begin{aligned}
& { }^{C-P} \mathbb{D}_{q}^{\alpha_{1}(p, q)} z(p, q)={ }^{1} \varepsilon_{\mathrm{Q}, n-\alpha_{1}(p, q), \omega, 0^{+}}^{-v} \frac{d^{n}}{d q^{n}}(z(p, q)), \\
& { }^{C-P} \mathbb{D}_{q}^{\alpha_{1}(p, q)} z(p, q)={ }^{2} \varepsilon_{\mathrm{Q}, n-\alpha_{1}(p, q), \omega, 0^{+}}^{-v} \frac{d^{n}}{d p^{n}}(z(p, q)),
\end{aligned}
$$

where $\mathfrak{R}_{(\mathrm{\varrho})}>0, \mathfrak{R}\left(\alpha_{1}(p, q)\right)>0, \varrho, \alpha_{1}(p, q), v, \omega \in \mathbb{C}$. Here, we consider $n=2$.
Lemma 3. Let $k>1$. Then, the following formula based on Definition 1 is established [63]:

$$
\begin{aligned}
& { }_{1}^{1} \varepsilon_{\mathrm{Q}, \mu(p, q), \omega, 0^{+}}^{v} q^{k-1}=\Gamma(k) q^{k+\mu(p, q)-1} e_{\mathrm{Q}, k+\mu(p, q)+1}^{v}(q), \\
& { }^{2} \varepsilon_{\mathrm{Q}, \mu(p, q), \omega, 0^{+}}^{v} p^{k-1}=\Gamma(k) p^{k+\mu(p, q)-1} e_{\mathrm{Q}, k+\mu(p, q)+1}^{v}(p) .
\end{aligned}
$$

Lemma 4. Let $k>2$. Then the following formula based on the Definition 2 and the Lemma 3 for $n=2$ are established:

$$
\begin{aligned}
& { }^{C-P} \mathbb{D}_{q}^{\alpha_{1}(p, q)} q^{k-1}=\Gamma(k) q^{k+\mu(p, q)-1} e_{\mathrm{Q}, k+\mu(p, q)+1}^{-v}(q), \\
& { }^{C-P} \mathbb{D}_{p}^{\alpha_{1}(p, q)} p^{k-1}=\Gamma(k) p^{k+\mu(p, q)-1} e_{\mathrm{Q}, k+\mu(p, q)+1}^{-v}(p) .
\end{aligned}
$$

Definition 5. The Chebyshev polynomials on $[-1,1]$ are defined as follows [64]:

$$
\begin{aligned}
T_{0}(p) & =1, T_{1}(p)=p, \\
T_{m+1}(p) & =2 p T_{m}(p)-T_{m-1}(p),
\end{aligned}
$$

where $T_{m}(p)=m \sum_{j=0}^{[m / 2]}(-1)^{j}\left(2^{m-2 j-1}(m-j-1) ! / j !(m-2 j) !\right.$ )$p^{m-2 j}$. The orthogonality manner for Chebyshev polynomials is given by

$$
\int_{-1}^{1} \Omega(p) T_{i}(p) T_{j}(p) d p= \begin{cases}\pi, & i=j=0 \\ \frac{\pi}{2}, & i=j \neq 0 \\ 0, & i \neq j\end{cases}
$$

where $\Omega(p)=1 / \sqrt{1-p^{2}}$ is a weight function for Chebyshev polynomials. Also, the shifted Chebyshev polynomials on $[0,1]$ are defined as follows:

$$
\begin{gathered}
\widehat{T}_{0}(q)=T_{0}(2 p-1)=1, \widehat{T}_{1}(q)=T_{1}(2 p-1)=q, \\
\underbrace{T_{m+1}(2 p-1)}_{\widehat{T}_{m+1}(q)}=\underbrace{2(2 p-1) T_{m}(2 p-1)-T_{m-1}(2 p-1)}_{2 q \widehat{T}_{m}(q)-\widehat{T}_{m-1}(q)},
\end{gathered}
$$

where $\widehat{T}_{m}(q)=m \sum_{j=0}^{m}(-1)^{m-j}\left(2^{2 j}(m+j-1) ! / 2 j !(m-j) !\right) q^{j}$. The orthogonality conditions for shifted Chebyshev polynomials are given by 


$$
\int_{0}^{1} \Omega^{*}(q) \widehat{T}_{i}(q) \widehat{T}_{j}(q) d q= \begin{cases}\frac{\pi}{2}, & i=j=0, \\ \frac{\pi}{4}, & i=j \neq 0, \\ 0, & i \neq j,\end{cases}
$$

where $\Omega^{*}(q)=1 / \sqrt{1-q^{2}}$ is a weight function.
Lemma 6. Let $\Psi(q)=\left[T \wedge_{0}(q), T \wedge_{l}(q), \cdots, T \wedge_{m}(q)\right]^{T}$. Then, the function $\Psi(q)$ has a matrix display as follows [64]:

$$
\Psi(q)=\mathbb{A} H_{m}(q),
$$

where $\mathbb{A}$ and $H(q)$ are defined by
Also, Lemma 6 is established for the function $\Psi(p)$.

\section{Approximate Function}

The approximation functions as $z(p, q), v(p, q)$ on $L^{2}([0,1]$ $\times[0,1])$ can be developed in shifted Chebyshev polynomials as follows:

$$
\begin{gathered}
z(p, q) \simeq z_{m}(p, q)=\sum_{i=0}^{m} \sum_{j=0}^{m} z_{i j} \widehat{T}_{i}(p) \widehat{T}_{j}(q)=\Psi^{T}(p) Z \Psi(q), \\
v(p, q) \simeq v_{m}(p, q)=\sum_{i=0}^{m} \sum_{j=0}^{m} v_{i j} \widehat{T}_{i}(p) \widehat{T}_{j}(q)=\Psi^{T}(p) V \Psi(q),
\end{gathered}
$$

where $Z=\left[z_{i j}\right]_{(m+1) \times(m+1)}, z_{i j}=\left\langle\widehat{T}_{i}(p),\left\langle z(p, q), \widehat{T}_{j}(q)\right\rangle\right\rangle$ and $V=\left[v_{i j}\right]_{(m+1) \times(m+1)}, \quad v_{i j}=\left\langle\widehat{T}_{i}(p),\left\langle v(p, q), \widehat{T}_{j}(q)\right\rangle\right\rangle$. By giving effect Caputo-Prabhakar derivative ${ }^{C-P} \mathbb{D}_{q}^{\alpha_{1}(p, q)}$ on Equation
(22), we obtain

$$
{ }^{C-P} \mathbb{D}_{q}^{\alpha_{1}(p, q)} z(p, q) \simeq{ }^{C-P} \mathbb{D}_{q}^{\alpha_{1}(p, q)} z_{m}(p, q)=\Psi^{T}(p) Z\left[{ }^{C-P} \mathbb{D}_{q}^{\alpha_{1}(p, q)} \Psi(q)\right],
$$

using Lemma 6, then from Equation (24), we have

$$
\begin{aligned}
{ }^{C-P} \mathbb{D}_{q}^{\alpha_{1}(p, q)} z_{m}(p, q) & =\Psi^{T}(p) Z\left[{ }^{C-P} \mathbb{D}_{q}^{\alpha_{1}(p, q)} \Psi(q)\right] \\
& =\Psi^{T}(p) Z \mathbb{A}\left[{ }^{C-P} \mathbb{D}_{q}^{\alpha_{1}(p, q)} H_{m}(p)\right] \\
& =\Psi^{T}(p) Z \mathbb{A}\left(\begin{array}{c}
{ }^{C-P} \mathbb{D}_{q}^{\alpha_{1}(p, q)_{1}} \\
C-P \mathbb{D}_{q}^{\alpha_{1}(p, q)_{q}} \\
\vdots \\
C-P \\
\mathbb{D}_{q}^{\alpha_{1}(p, q)_{q^{m}}}
\end{array}\right),
\end{aligned}
$$

applying Lemma 4 on Equation (25), we have 


$$
\begin{aligned}
& { }^{C-P} \mathbb{D}_{q}^{\alpha_{1}(p, q)} z_{m}(p, q)=\Psi^{T}(p) Z \mathbb{A}\left(\begin{array}{c}
C-P \mathbb{D}_{q}^{\alpha_{1}(p, q)_{1}} \\
{ }^{C-P} \mathbb{D}_{q}^{\alpha_{1}(p, q)_{q}} \\
\vdots \\
C-P \mathbb{D}_{q}^{\alpha_{1}(p, q)_{q^{m}}}
\end{array}\right)=\Psi^{T}(p) Z \mathbb{A}\left(\begin{array}{c}
0 \\
0 \\
\vdots \\
\Gamma(m+1) q^{m-\alpha_{1}(p, q) e_{e, m-\alpha_{1}(p, q)+1(q)}^{-\nu}}
\end{array}\right) \\
& =\Psi^{T}(p) Z A\left(\begin{array}{cccc}
0 & 0 & \cdots & 0 \\
0 & \Gamma(2) q^{1-\alpha_{1}(p, q) \mathrm{e}_{\mathrm{Q}, 2-\alpha_{1}(p, q)(q)}^{-\nu}} & \cdots & 0 \\
\vdots & \vdots & \ddots & \vdots \\
0 & 0 & 0 & \Gamma(m+1) q^{m-\alpha_{1}(p, q) \mathrm{e}_{\mathrm{Q}, m-\alpha_{1}(p, q)+1}^{-v}(q)}
\end{array}\right) \times\left(\begin{array}{c}
1 \\
q \\
\vdots \\
q^{m}
\end{array}\right) \\
& =\Psi^{T}(p) Z \operatorname{AM}_{1} H_{m}(q)=\Psi^{T}(p) Z \operatorname{AM}_{1} \mathbb{A}^{-1} \Psi(q), \\
& { }^{C-P} \mathbb{D}_{q}^{\alpha_{1}(p, q)} z(p, q) \cong{ }^{C-P} \mathbb{D}_{q}^{\alpha_{1}(p, q)} z_{m}(p, q)=\Psi^{T}(p) Z \mathbb{A M}_{1} \mathbb{A}^{-1} \Psi(q),
\end{aligned}
$$

where

$$
\mathbb{M}_{1}=\left(\begin{array}{cccc}
0 & 0 & \cdots & 0 \\
0 & \Gamma(2) q^{1-\alpha_{1}(p, q)} e_{\rho, 2-\alpha_{1}(p, q)}^{-v}(q) & \cdots & 0 \\
\vdots & \vdots & \ddots & \vdots \\
0 & 0 & 0 & \Gamma(m+1) q^{m-\alpha_{1}(p, q)} e_{\rho, m-\alpha_{1}(p, q)+1}^{-v}(q)
\end{array}\right)
$$

With a similar process as Equation (26), these results are easily achieved:

$$
{ }^{C-P} \mathbb{D}_{q}^{\alpha_{2}(p, q)} v_{m}(p, q)=\Psi^{T}(p) V \mathbb{A M}_{2} \mathbb{A}^{-1} \Psi(q),
$$
where

$$
\begin{aligned}
& \mathbb{M}_{2}=\left(\begin{array}{cccc}
0 & 0 & \cdots & 0 \\
0 & \Gamma(2) q^{1-\alpha_{2}(p, q)} e_{\rho, 2-\alpha_{2}(p, q)}^{-v}(q) & \cdots & 0 \\
\vdots & \vdots & \ddots & \vdots \\
0 & 0 & 0 & \Gamma(m+1) q^{m-\alpha_{2}(p, q)} e_{\rho, m-\alpha_{2}(p, q)+1}^{-v}(q)
\end{array}\right) \\
& { }^{C-P} \mathbb{D}_{p}^{\alpha_{1}(p, q)} z_{m}(p, q)={ }^{C-P} \mathbb{D}_{p}^{\alpha_{1}(p, q)}\left[1, p, \cdots, p^{m}\right] \mathbb{A}^{T} Z \Psi(q)=\left[0,0, \cdots, \Gamma(m+1) p^{m-\alpha_{1}(p, q)} e_{\rho, m-\alpha_{1}(p, q)+1}^{-v}(p)\right] \mathbb{A}^{T} Z \Psi(q) \\
& =\left[1, p, \cdots, p^{m}\right]\left(\begin{array}{cccc}
0 & 0 & \cdots & 0 \\
0 & \Gamma(2) p^{1-\alpha_{1}(p, q)} e_{\rho, 2-\alpha_{1}(p, q)}^{-v}(p) & \cdots & 0 \\
\vdots & \vdots & \ddots & \vdots \\
0 & 0 & 0 & \Gamma(m+1) p^{m-\alpha_{1}(p, q)} e_{\rho, m-\alpha_{1}(p, q)+1}^{-v}(p)
\end{array}\right) \\
& =H_{m}^{T}(p) \mathbb{M}_{3} \mathbb{A}^{T} V \Psi(q)=\Psi^{T}(p)\left(\mathbb{A}^{-1}\right)^{T} \mathbb{M}_{3} \mathbb{A}^{T} V \Psi(q),
\end{aligned}
$$


where

$$
\begin{aligned}
& \mathbb{M}_{3}=\left(\begin{array}{cccc}
0 & 0 & \cdots & 0 \\
0 & \Gamma(2) p^{1-\alpha_{1}(p, q)} e_{\rho, 2-\alpha_{1}(p, q)}^{-v}(p) & \cdots & 0 \\
\vdots & \vdots & \ddots & \vdots \\
0 & 0 & 0 & \Gamma(m+1) p^{m-\alpha_{1}(p, q)} e_{\rho, m-\alpha_{1}(p, q)+1}^{-v}(p)
\end{array}\right), \\
& { }^{C-P} \mathbb{D}_{p}^{\alpha_{2}(p, q)} v_{m}(p, q)={ }^{C-P} \mathbb{D}_{p}^{\alpha_{2}(p, q)}\left[1, p, \cdots, p^{m}\right] \mathbb{A}^{T} V \Psi(q)=\left[0,0, \cdots, \Gamma(m+1) p^{m-\alpha_{1}(p, q)} e_{\rho, m-\alpha_{1}(p, q)+1}^{-v}(p)\right] A^{T} V \Psi(q) \\
& =\left[1, p, \cdots, p^{m}\right]\left(\begin{array}{cccc}
0 & 0 & \cdots & 0 \\
0 & \Gamma(2) p^{1-\alpha_{2}(p, q)} e_{\rho, 2-\alpha_{2}(p, q)}^{-v}(p) & \cdots & 0 \\
\vdots & \vdots & \ddots & \vdots \\
0 & 0 & 0 & \Gamma(m+1) p^{m-\alpha_{2}(p, q)} e_{\rho, m-\alpha_{2}(p, q)+1}^{-v}(p)
\end{array}\right) \\
& =H_{m}^{T}(p) \mathbb{M}_{4} \mathbb{A}^{T} V \Psi(q)=\Psi^{T}(p)\left(\mathbb{A}^{-1}\right)^{T} \mathbb{M}_{4} \mathbb{A}^{T} V \Psi(q)
\end{aligned}
$$

where

$$
\mathbb{M}_{4}=\left(\begin{array}{cccc}
0 & 0 & \cdots & 0 \\
0 & \Gamma(2) p^{1-\alpha_{2}(p, q)} e_{\rho, 2-\alpha_{2}(p, q)}^{-v}(p) & \cdots & 0 \\
\vdots & \vdots & \ddots & \vdots \\
0 & 0 & 0 & \Gamma(m+1) p^{m-\alpha_{2}(p, q)} e_{\rho, m-\alpha_{2}(p, q)+1}^{-v}(p)
\end{array}\right) .
$$

3.1. Description of Convergence. Let $z(p, q), v(p, q)$ be the exact solutions of Equations (3) and $z_{m}(p, q), v_{m}(p, q)$ be the numerical solutions of Equations (3) which are introduced in (22)-(23), then we show that as $\longrightarrow \infty$, we have $\left\|z-z_{m}\right\|^{2} \longrightarrow 0$ and $\left\|v-v_{m}\right\|^{2} \longrightarrow 0$. For this purpose, we show that the sequences $z_{m}(p, q), v_{m}(p, q)$ are a Cauchy sequence in Hilbert space $L^{2}([a, b] \times[0, T])$. Then, for $\widehat{m}>$ $m$, we obtain

$$
\begin{aligned}
\left\|z_{\hat{m}}-z_{m}\right\|^{2} & =\left\|\sum_{i=0}^{\hat{m}} \sum_{j=0}^{\hat{m}} z_{i j} \widehat{T}_{i}(p) \widehat{T}_{j}(q)-\sum_{i=0}^{m} \sum_{j=0}^{m} z_{i j} \widehat{T}_{i}(p) \widehat{T}_{j}(q)\right\|^{2} \\
& =\left\|\sum_{i=m+1}^{\hat{m}} \sum_{j=m+1}^{\hat{m}} z_{i j} \widehat{T}_{i}(p) \widehat{T}_{j}(q)\right\|^{2} \\
& =\left\langle\sum_{i=m+1}^{\hat{m}} \sum_{j=m+1}^{\hat{m}} z_{i j} \widehat{T}_{i}(p) \widehat{T}_{j}(q), \sum_{l=m+1}^{\hat{m}} \sum_{k=m+1}^{\hat{m}} z_{l k} \widehat{T}_{l}(p) \widehat{T}_{k}(q)\right\rangle \\
& =\sum_{i=m+1}^{\hat{m}} \sum_{j=m+1}^{\hat{m}} \sum_{l=m+1}^{\hat{m}} \sum_{k=m+1}^{\hat{m}} z_{i j} \bar{z}_{l k}\left\langle\widehat{T}_{i}(p) \widehat{T}_{j}(q), \widehat{T}_{x}(p) \widehat{T}_{t}(q)\right\rangle \\
& =\frac{\pi^{2}}{16} \sum_{i=m+1}^{\hat{m}} \sum_{j=m+1}^{\hat{m}}\left|z_{i j}\right|^{2} .
\end{aligned}
$$

From Bessel's inequality, we obtain that the series is presented in (33) as $\longrightarrow$ oand $\widehat{m} \longrightarrow \infty$ is convergent, then $\left\|z_{\widehat{m}}-z_{m}\right\|^{2} \longrightarrow 0 .\left\|z_{\widehat{m}}-z_{m}\right\|^{2} \longrightarrow 0$ show that the sequence $z_{m}$ is a Cauchy sequence in Hilbert space $L^{2}([a, b]$ $\times[0, T])$ and it converges to say $z_{1}(p, q)$. Now, we show $z$ $=z_{1}(p, q)$, then

$$
\begin{aligned}
\langle z & \left.-z_{1}(p, q), \widehat{T}_{i}(p) \widehat{T}_{j}(q)\right\rangle \\
& =\left\langle z, \widehat{T}_{i}(p) \widehat{T}_{j}(q)\right\rangle-\left\langle z_{1}(p, q), \widehat{T}_{i}(p) \widehat{T}_{j}(q)\right\rangle \\
& =\lim _{m \longrightarrow \infty}\left\langle z_{\widehat{m}}, \widehat{T}_{i}(p) \widehat{T}_{j}(q)\right\rangle-z_{i j}=z_{i j}-z_{i j}=0,
\end{aligned}
$$

where $z_{i j}=\int_{0}^{T} \int_{a}^{b} z(p, q) \widehat{T}_{i}(p) \widehat{T}_{j}(q) \omega^{*}(p) \omega^{*}(q) d p d q$. Then, $\sum_{i=0}^{m} \sum_{j=0}^{m} z_{i j} \widehat{T}_{i}(p) \widehat{T}_{j}(q)$ converges to $z(p, q), v(p, q)$ as $m$ $\longrightarrow \infty$. The same process can be considered for the sequence $v_{m}$.

3.2. Error Analysis. Suppose that $z(p, q), v(p, q) \in C(\Omega)$, $\Omega=[0,1] \times[0,1]$ be the exact solutions, respectively. If $z_{m}(p, q)=\Lambda^{\prime} \times \Lambda, v_{m}(p, q)=\Lambda^{\prime} \times \Lambda$ be the best numerical solution to $z(p, q)$ that $\Lambda=\operatorname{Span}\left\{\widehat{T}_{0}(q), \widehat{T}_{1}(q), \cdots, \widehat{T}_{m}(q)\right\}$, 
$\Lambda^{\prime} \in \operatorname{Span}\left\{\widehat{T}_{0}(p), \widehat{T}_{1}(p), \cdots, \widehat{T}_{m}(p)\right\}$. We define $\mathscr{E}_{m}$ which is the error as follows:

$$
\begin{aligned}
{ }^{C-P} \mathbb{D}_{q}^{\alpha_{1}(p, q)} z(p, q)-{ }^{C-P} \mathbb{D}_{p}^{\alpha_{1}(p, q)} z(p, q)+\theta^{2} \sin (z-v)-h_{1}(p, q) \\
={ }^{C-P} \mathbb{D}_{q}^{\alpha_{1}(p, q)} z_{m}(p, q)-{ }^{C-P} \mathbb{D}_{p}^{\alpha_{1}(p, q)} z_{m}(p, q)+\theta^{2} \sin \left(z_{m}-v_{m}\right) \\
\quad-h_{1}(p, q)+\mathscr{E}_{m} .
\end{aligned}
$$

Then, from Eq. (35), we have:

$$
\begin{aligned}
\left\|\mathscr{E}_{m}\right\|_{L^{2}(\Omega)}= & \|^{C-P} \mathbb{D}_{q}^{\alpha_{1}(p, q)}\left(z_{m}-z\right)-{ }^{C-P} \mathbb{D}_{p}^{\alpha_{1}(p, q)}\left(z_{m}-z\right) \\
& +\theta^{2}\left[\sin \left(z_{m}-v_{m}\right)-\sin (z-v)\right] \|_{L^{2}(\Omega)} \\
\leq & \left\|^{C-P} \mathbb{D}_{q}^{\alpha_{1}(p, q)}\left(z_{m}-z\right)\right\|_{L^{2}(\Omega)}+\left\|^{C-P} \mathbb{D}_{p}^{\alpha_{1}(p, q)}\left(z_{m}-z\right)\right\|_{L^{2}(\Omega)} \\
& +\left\|\theta^{2}\left[\sin \left(z_{m}-v_{m}\right)-\sin (z-v)\right]\right\|_{L^{2}(\Omega)} \\
\leq & \left\|^{C-P} \mathbb{D}_{q}^{\alpha_{1}(p, q)}\left(z_{m}-z\right)\right\|_{L^{2}(\Omega)}+\left\|^{C-P} \mathbb{D}_{p}^{\alpha_{1}(p, q)}\left(z_{m}-z\right)\right\|_{L^{2}(\Omega)} \\
& +\theta^{2}\left\|\sin \left(z_{m}-v_{m}\right)\right\|_{L^{2}(\Omega)}+\theta^{2}\|\sin (z-v)\|_{L^{2}(\Omega)} .
\end{aligned}
$$

To get Equation (36), we define

$$
\begin{aligned}
& \Delta_{m}^{\alpha(p, q), \dot{\alpha}(p, q)}(p, q) \\
& \quad=\sum_{i=0}^{m} \sum_{j=0}^{m} \frac{p^{i \alpha(p, q)} q^{j \alpha}(p, q)}{\Gamma(i \alpha(p, q)+1) \Gamma\left(j \alpha^{\prime}(p, q)+1\right)} \mathbb{D}_{p}^{i \alpha(p, q)} \\
& \left.\cdot\left({ }^{C-P} \mathbb{D}_{q}^{j \alpha}(p, q) z(p, q)\right)\right|_{(0,0)} .
\end{aligned}
$$

From the multivariable Taylor definition and the Generalized Taylor's formula in $[65,66]$, we have

$$
\begin{aligned}
& \left|z(p, q)-\Delta_{m}^{\alpha(p, q), \alpha^{\prime}(p, q)}(p, q)\right| \\
& \quad \leq \frac{p^{(m+1) \alpha(p, q)} q^{(m+1) \alpha^{\prime}(p, q)}}{\Gamma((m+1) \alpha(p, q)+1) \Gamma\left((m+1) \alpha^{\prime}(p, q)+1\right)} Y_{m}^{\alpha(p, q), \alpha^{\prime}(p, q)},
\end{aligned}
$$

where $Y_{m}^{\alpha(p, q) ! \alpha(p, q)}=\left.\sup _{(p, q) \in \Omega}\right|^{C-P} \mathbb{D}_{p}^{i \alpha(p, q)}\left({ }^{C-P} \mathbb{D}_{q}^{j \alpha(p, q)} z(p, q)\right) \mid$. By using Lemma 4, we have

$$
\begin{aligned}
\mid{ }^{C-P} & \mathbb{D}_{q}^{\alpha_{1}(p, q)} z(p, q)-{ }^{C-P} \mathbb{D}_{q}^{\alpha_{1}(p, q)} \Delta_{m}^{\alpha(p, q) \jmath \alpha(p, q)}(p, q) \mid \\
\leq & \frac{p^{(m+1) \alpha(p, q)} q^{(m+1) \alpha^{\prime}(p, q)-\alpha_{1}(p, q)} e_{\rho,(m+1) \alpha^{\prime(p, q)-\alpha_{1}(p, q)+1}}^{-v}(q)}{\Gamma((m+1) \alpha(p, q)+1)} \\
& \times Y_{m}^{\alpha(p, q)\rfloor \alpha(p, q)},
\end{aligned}
$$

$$
\begin{aligned}
\mid{ }^{C-P} & \mathbb{D}_{p}^{\alpha_{1}(p, q)} z(p, q)-{ }^{C-P} \mathbb{D}_{p}^{\alpha_{1}(p, q)} \Delta_{m}^{\alpha(p, q) ! \alpha(p, q)}(p, q) \mid \\
\leq & \frac{q^{(m+1) \alpha^{\prime}(p, q)} p^{(m+1) \alpha^{\prime}(p, q)-\alpha_{1}(p, q)} e_{\rho,(m+1) \alpha^{\prime}(p, q)-\alpha_{1}(p, q)+1}^{-v}(p)}{\Gamma\left((m+1) \alpha^{\prime}(p, q)+1\right)} \\
& \times Y_{m}^{\alpha(p, q) ! \alpha(p, q)} .
\end{aligned}
$$

Then,

$$
\begin{aligned}
\left\|^{C-P} \mathbb{D}_{q}^{\alpha_{1}(p, q)}\left(z_{m}-z\right)\right\|_{L^{2}(\Omega)}^{2} & \leq\left\|^{C-P} \mathbb{D}_{q}^{\alpha_{1}(p, q)} z(p, q)-{ }^{C-P} \mathbb{D}_{q}^{\alpha_{1}(p, q)} \Delta_{m}^{\alpha(p, q), \dot{\alpha}(p, q)}(p, q)\right\|_{L^{2}(\Omega)}^{2} \\
& \leq \int_{0}^{1} \int_{0}^{1}\left(\frac{x^{(m+1) \alpha(p, q)} t^{(m+1) \alpha^{\prime}(p, q)-\alpha_{1}(p, q)} e_{\rho,(m+1) \alpha^{\prime}(p, q)-\alpha_{1}(p, q)+1}^{-v}(t)}{\Gamma((m+1) \alpha(p, q)+1)} Y_{m}^{\alpha(p, q), \dot{\alpha}(p, q)}\right)^{2} d t d x
\end{aligned}
$$

According to the definition $e_{\rho,(m+1) \alpha^{\prime}(p, q)-\alpha_{1}(p, q)+1}^{-v}(t)$, we have

$e_{\rho,(m+1) \alpha^{\prime}(p, q)-\alpha_{1}(p, q)+1}^{-v}(t)=t^{(m+1) \alpha^{\prime}(p, q)-\alpha_{1}(p, q)} E_{\rho,(m+1) \alpha^{\prime}(p, q)-\alpha_{1}(p, q)+1}^{v}\left(\omega t^{\rho}\right)$.
From $[16,67,68]$, the Prabhakar function is convergent and the convergent boundary for its $M$ is considered, then we obtain

$$
\left\|{ }^{C-P} \mathbb{D}_{q}^{\alpha_{1}(p, q)}\left(z_{m}-z\right)\right\|_{L^{2}(\Omega)}^{2} \leq \frac{M^{2}\left(Y_{m}^{\alpha(p, q)\lrcorner \alpha(p, q)}\right)^{2}}{(2(m+1) \alpha(p, q)+1)\left(4\left((m+1) \alpha^{\prime}(p, q)-\alpha_{1}(p, q)\right)+1\right)(\Gamma((m+1) \alpha(p, q)+1))^{2}},
$$


also

$$
\begin{aligned}
\left\|^{C-P} \mathbb{D}_{p}^{\alpha_{1}(p, q)}\left(z_{m}-z\right)\right\|_{L^{2}(\Omega)}^{2} & \leq\left\|^{C-P} \mathbb{D}_{p}^{\alpha_{1}(p, q)} z(p, q)-^{C-P} \mathbb{D}_{p}^{\alpha_{1}(p, q)} \Delta_{m}^{\alpha(p, q)) \alpha(p, q)}(p, q)\right\|_{L^{2}(\Omega)}^{2} \\
& \leq \int_{0}^{1} \int_{0}^{1}\left(\frac{t^{(m+1) \alpha^{\prime}(p, q)} x^{(m+1) \alpha^{\prime}(p, q)-\alpha_{1}(p, q)} e_{\rho,(m+1) \alpha^{\prime}(p, q)-\alpha_{1}(p, q)+1}^{-v}(x)}{\Gamma\left((m+1) \alpha^{\prime}(p, q)+1\right)} Y_{m}^{\alpha(p, q)) \alpha(p, q)}\right)^{2} d t d x,
\end{aligned}
$$

from

$$
\left|E_{\rho,(m+1) \alpha^{\prime}(p, q)-\alpha_{1}(p, q)+1}^{v}\left(\omega x^{\rho}\right)\right| \leq M_{1}
$$

$e_{\rho,(m+1) \alpha^{\prime}(p, q)-\alpha_{1}(p, q)+1}^{-v}(x)=x^{(m+1) \alpha^{\prime}(p, q)-\alpha_{1}(p, q)} E_{\rho,(m+1) \alpha^{\prime}(p, q)-\alpha_{1}(p, q)+1}^{v}\left(\omega x^{\rho}\right), \quad$ we have

$$
\left\|^{C-P} \mathbb{D}_{p}^{\alpha_{1}(p, q)}\left(z_{m}-z\right)\right\|_{L^{2}(\Omega)}^{2} \leq \frac{M_{1}^{2}\left(Y_{m}^{\alpha(p, q)) \alpha(p, q)}\right)^{2}}{\left(2(m+1) \alpha^{\prime}(p, q)+1\right)\left(4\left((m+1) \alpha^{\prime}(p, q)-\alpha_{1}(p, q)\right)+1\right)\left(\Gamma\left((m+1) \alpha^{\prime}(p, q)+1\right)\right)^{2}} .
$$

So by placing Equations (42) and (45) into (36), we have

$$
\begin{aligned}
\left\|\mathscr{C}_{m}\right\|_{L^{2}(\Omega)} \leq & \frac{M Y_{m}^{\alpha(p, q)) \alpha(p, q)}}{\sqrt{(2(m+1) \alpha(p, q)+1)\left(4\left((m+1) \alpha^{\prime}(p, q)-\alpha_{1}(p, q)\right)+1\right)}(\Gamma((m+1) \alpha(p, q)+1))} \\
& +\frac{M_{1} Y_{m}^{\alpha(p, q)) \alpha(p, q)}}{\sqrt{\left(2(m+1) \alpha^{\prime}(p, q)+1\right)\left(4\left((m+1) \alpha^{\prime}(p, q)-\alpha_{1}(p, q)\right)+1\right)}\left(\Gamma\left((m+1) \alpha^{\prime}(p, q)+1\right)\right)}+\theta^{2}\left(R+R_{1}\right),
\end{aligned}
$$

where $R=\sup _{(p, q) \in \Omega}|\sin (z-v)|$ and $R_{1}=\sup _{(p, q) \in \Omega} \mid \sin$ $\left(z_{m}-v_{m}\right) \mid$. With a similar process, the following inequal- ity can be considered for the function $\mathscr{E}_{m}^{\prime}$ that is defined as follows:

$$
\begin{aligned}
&{ }^{C-P} \mathbb{D}_{q}^{\alpha_{2}(p, q)} v(p, q)-\xi^{2 C-P} \mathbb{D}_{p}^{\alpha_{2}(p, q)} v(p, q)-\sin (z-v)-h_{1}(p, q) \\
&={ }^{C-P} \mathbb{D}_{q}^{\alpha_{2}(p, q)} v_{m}(p, q)-\xi^{2 C-P} \mathbb{D}_{p}^{\alpha_{2}(p, q)} v_{m}(p, q)-\sin \left(z_{m}-v_{m}\right)-h_{1}(p, q)+\mathscr{E}_{m}^{\prime}, \\
&\left\|\mathscr{E}^{\prime}{ }_{m}\right\|_{L^{2}(\Omega)} \leq \frac{M Y_{m}^{\alpha(p, q)) \alpha(p, q)}}{\sqrt{(2(m+1) \alpha(p, q)+1)\left(4\left((m+1) \alpha^{\prime}(p, q)-\alpha_{2}(p, q)\right)+1\right)}(\Gamma((m+1) \alpha(p, q)+1))} \\
&+\xi^{2} \frac{M_{1} Y_{m}^{\alpha(p, q) s \alpha(p, q)}}{\sqrt{\left(2(m+1) \alpha^{\prime}(p, q)+1\right)\left(4\left((m+1) \alpha^{\prime}(p, q)-\alpha_{2}(p, q)\right)+1\right)}\left(\Gamma\left((m+1) \alpha^{\prime}(p, q)+1\right)\right)}+R+R_{1} .
\end{aligned}
$$




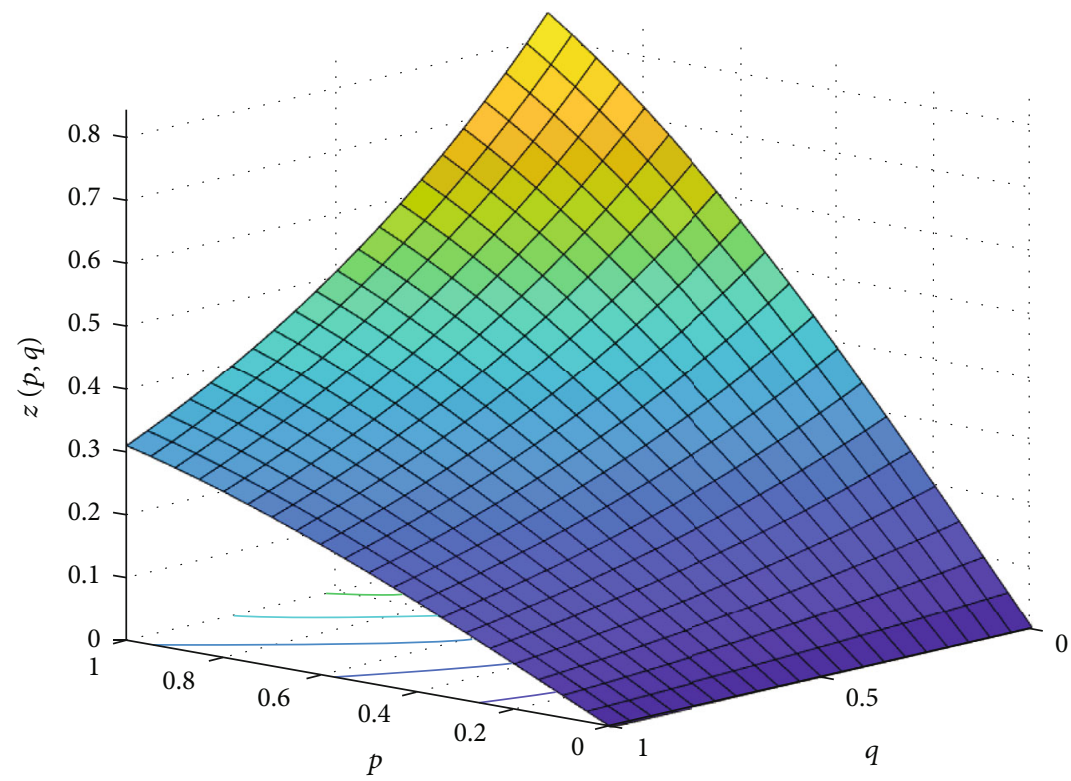

(a)

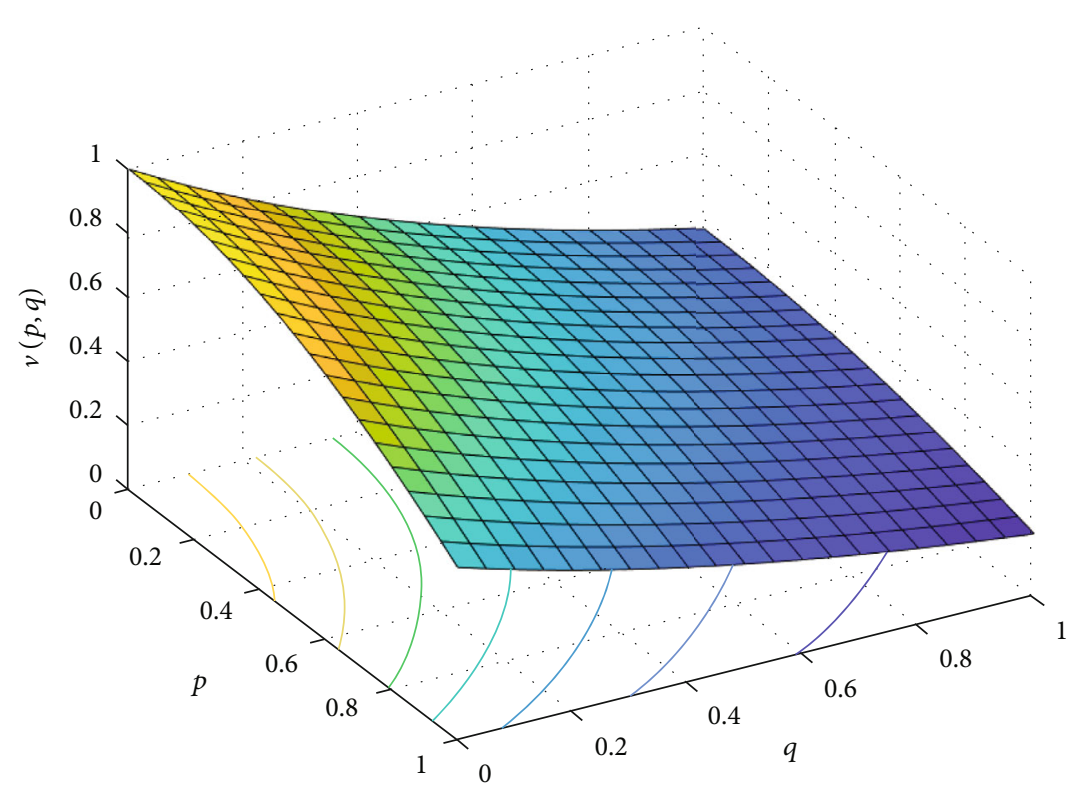

(b)

FIGURE 1: Numerical solution of $z(p, q), v(p, q)$ with $\rho=\omega=v=1$ for Example 7 when $m=6$.

\section{Description of the Suggested Method}

This section describes the suggested method to obtain solutions of the equations considered in (3) with the conditions (6) and (8). Then, we put relations (26)-(30) into Equation (3), and the following results are obtained:

$$
\begin{gathered}
\Psi^{T}(p) Z \mathbb{A M}_{1} \mathbb{A}^{-1} \Psi(q)-\Psi^{T}(p)\left(\mathbb{A}^{-1}\right)^{T} \mathbb{M}_{3} \mathbb{A}^{T} V \Psi(q) \\
+\theta^{2} \Psi^{T}(p) \sin (Z-V) \Psi(q)-h_{1}(p, q) \simeq 0,
\end{gathered}
$$

$$
\Psi^{T}(p)\left[Z \operatorname{ZMM}_{1} \mathbb{A}^{-1}-\left(\mathbb{A}^{-1}\right)^{T} \mathbb{M}_{3} \mathbb{A}^{T} V+\theta^{2} \sin (Z-V)\right] \Psi(q)-h_{1}(p, q) \simeq 0,
$$

$$
\begin{aligned}
& \Psi^{T}(p) V \operatorname{VAM}_{2} \mathbb{A}^{-1} \Psi(q)-\xi^{2} \Psi^{T}(p)\left(\mathbb{A}^{-1}\right)^{T} \mathbb{M}_{4} \mathbb{A}^{T} V \Psi(q) \\
& \quad-\Psi^{T}(p) \sin (Z-V) \Psi(q)-h_{2}(p, q) \\
& \simeq 0, \Psi^{T}(p)\left[V A M_{2} \mathbb{A}^{-1}-\xi^{2}\left(\mathbb{A}^{-1}\right)^{T} \mathbb{M}_{4} \mathbb{A}^{T} V-\sin (Z-V)\right] \Psi(q) \\
& \quad \times-h_{2}(p, q) \simeq 0
\end{aligned}
$$




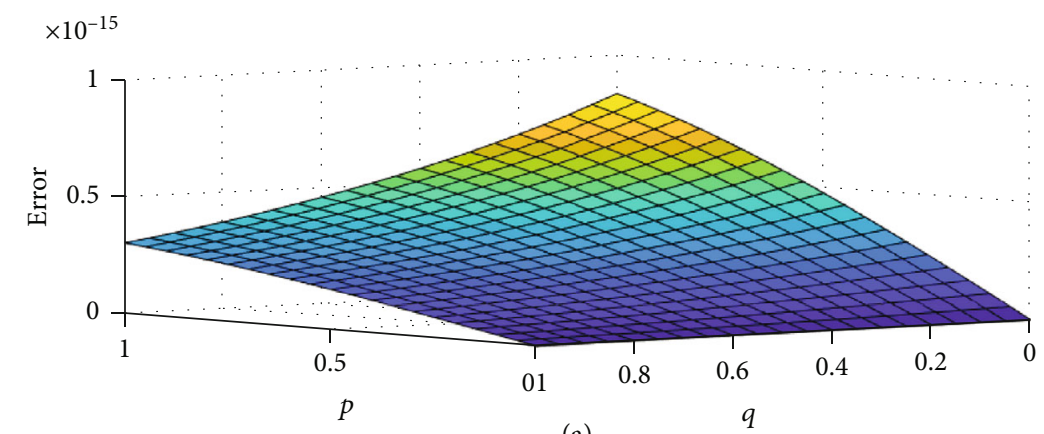

(a)

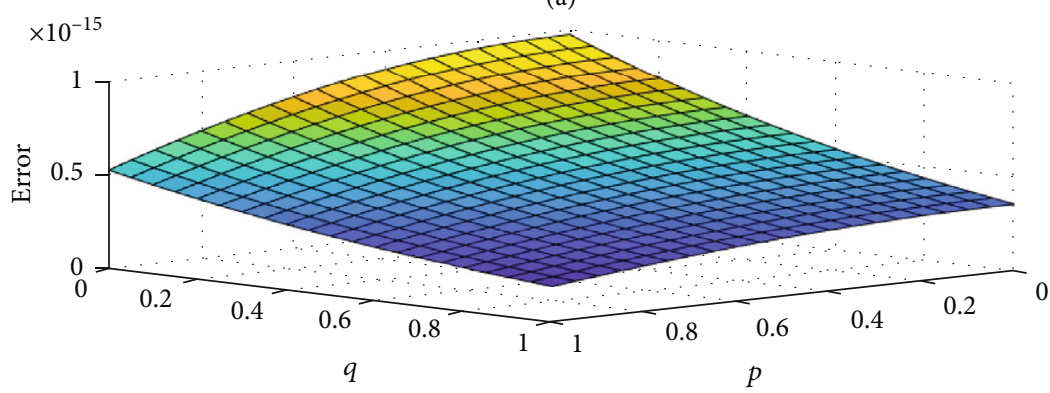

(b)

Figure 2: Charts of absolute error $z$ (a) and $v(\mathrm{~b})$ with $\rho=\omega=v=1$ for Example 7 when $m=6$.

where $\sin (z-v) \simeq \sin \left(z_{m}-v_{m}\right)=\sum_{i=0}^{m} \sum_{j=0}^{m} \sin \left(z_{i j}-v_{i j}\right)$ $\widehat{T}_{i}(p) \widehat{T}_{j}(q)=\Psi^{T}(p) \sin (Z-V) \Psi(q)$. Considering the formulas $l_{k}(p) \simeq \Psi^{T}(p) L_{k}, k=0,1,2,3$ and $f_{k}(q) \simeq F_{k}^{T} \Psi(q), k$ $=0,1,2,3, F_{k}, L_{k}$ are known. Then, by substituting functions $l_{k}(p), f_{k}(q), k=0,1,2,3$, and Equations (22) and (23) in the conditions introduced in Equations (6) and (4), we obtain

$\Psi^{T}(p) Z \Psi(0)-\Psi^{T}(p) L_{0} \simeq 0, \Psi^{T}(p) Z \mathbb{A M}_{1} \mathbb{A}^{-1} \Psi(0)-\Psi^{T}(p) L_{1} \simeq 0$

$\Psi^{T}(p) V \Psi(0)-\Psi^{T}(p) L_{2} \simeq 0, \Psi^{T}(p) V A \mathbb{M}_{2} \mathbb{A}^{-1} \Psi(0)-\Psi^{T}(p) L_{3} \simeq 0$,

$$
\Psi^{T}(a) Z \Psi(q)-F_{0}^{T} \Psi(q) \simeq 0, \Psi^{T}(b) Z \Psi(q)-F_{1}^{T} \Psi(q) \simeq 0,
$$

$\Psi^{T}(a) V \Psi(q)-F_{2}^{T} \Psi(q) \simeq 0, \Psi^{T}(b) V \Psi(q)-F_{3}^{T} \Psi(q) \simeq 0$.

To find solutions of problems (3) which are $z$, $v$, we solve the Equations (49)-(50) corresponding to (51)-(54) in collocation points to obtain functions $Z, V$.

\section{Numerical Examples}

In this section, we show the numerical results of the suggested method on four examples to illustrate the efficiency and accuracy of our scheme. Here, obtained numerical results of some test problems are shown to illustrate the computational efficiency and applicability of the reported method. All numerical examples are done by applying
TABLE 1: The absolute error for different values $m$ for Example 7 at various points $(p, q)$.

\begin{tabular}{lcccc}
\hline$(p, q)$ & $m=6$ & $m=7$ & $m=8$ & $m=9$ \\
\hline$(0.1,0.1)$ & $8.9329 e-17$ & $6.2530 e-16$ & $7.1463 e-16$ & $8.0396 e-16$ \\
$(0.2,0.2)$ & $1.6084 e-16$ & $1.1259 e-15$ & $1.2867 e-15$ & $1.4476 e-15$ \\
$(0.3,0.3)$ & $2.1649 e-16$ & $1.5154 e-15$ & $1.7319 e-15$ & $1.9484 e-15$ \\
$(0.4,0.4)$ & $2.5813 e-16$ & $1.8069 e-15$ & $2.0650 e-15$ & $2.3232 e-15$ \\
$(0.5,0.5)$ & $2.8755 e-16$ & $2.0128 e-15$ & $2.3004 e-15$ & $2.5880 e-15$ \\
$(0.6,0.6)$ & $3.0643 e-16$ & $2.1450 e-15$ & $2.4515 e-15$ & $2.7579 e-15$ \\
$(0.7,0.7)$ & $3.1635 e-16$ & $2.2144 e-15$ & $2.5308 e-15$ & $2.8471 e-15$ \\
$(0.8,0.8)$ & $3.1874 e-16$ & $2.2312 e-15$ & $2.5499 e-15$ & $2.8687 e-15$ \\
$(0.9,0.9)$ & $3.1493 e-16$ & $2.2045 e-15$ & $2.5195 e-15$ & $2.8344 e-15$ \\
$(1,1)$ & $3.0612 e-16$ & $2.1428 e-15$ & $2.4489 e-15$ & $2.7550 e-15$ \\
\hline
\end{tabular}

MATLAB (R2020b) software. Also, the convergence order ( $C$-order) of the depicted method is calculated by the following relation:

$$
C \text {-order }=\frac{\left|\log \left(E_{1} / E_{2}\right)\right|}{\log \left(\left(m_{2}+1\right)\left(m_{2}+1\right) /\left(m_{1}+1\right)\left(m_{1}+1\right)\right)} .
$$

Here, $E_{1}$ and $E_{2}$ show the first and second values of the maximum absolute error (MAE) provided by the proposed method, respectively. Moreover, $\left(m_{i}+1\right)\left(m_{i}+1\right)$ for $i=1,2$ are the number of the basis functions used in the first and second implementations, respectively. 
TABle 2: The absolute error for different values $m$ for Example 7 at various points $(p, q)$.

\begin{tabular}{|c|c|c|c|c|}
\hline \multirow{2}{*}{$(p, q)$} & \multicolumn{4}{|c|}{$\left|v_{m}-v\right|$} \\
\hline & $m=6$ & $m=7$ & $m=8$ & $m=9$ \\
\hline$(0.1,0.1)$ & $8.9031 e-16$ & $6.2322 e-15$ & $7.1225 e-15$ & $8.0128 e-15$ \\
\hline$(0.2,0.2)$ & $7.9349 e-16$ & $5.5544 e-15$ & $6.3479 e-15$ & $7.1414 e-15$ \\
\hline$(0.3,0.3)$ & $6.9986 e-16$ & $4.8990 e-15$ & $5.5989 e-15$ & $6.2988 e-15$ \\
\hline$(0.4,0.4)$ & $6.1054 e-16$ & $4.2738 e-15$ & $4.8843 e-15$ & $5.4949 e-15$ \\
\hline$(0.5,0.5)$ & $5.2636 e-16$ & $3.6845 e-15$ & $4.2109 e-15$ & $4.7373 e-15$ \\
\hline$(0.6,0.6)$ & $4.4792 e-16$ & $3.1354 e-15$ & $3.5833 e-15$ & $4.0312 e-15$ \\
\hline$(0.7,0.7)$ & $3.7558 e-16$ & $2.6291 e-15$ & $3.0047 e-15$ & $3.3803 e-15$ \\
\hline$(0.8,0.8)$ & $3.09572 e-16$ & $2.1670 e-15$ & $2.4765 e-15$ & $2.7861 e-15$ \\
\hline$(0.9,0.9)$ & $2.4991 e-16$ & $1.7494 e-15$ & $1.9993 e-15$ & $2.2492 e-15$ \\
\hline$(1,1)$ & $1.9655 e-16$ & $1.3759 e-15$ & $1.5724 e-15$ & $1.7690 e-15$ \\
\hline
\end{tabular}

Example 7. We consider the following coupled nonlinear variable-order time-fractional sine-Gordon equations:

$$
\begin{gathered}
{ }^{C-P} \mathbb{D}_{q}^{\alpha_{1}(p, q)} z(p, q){ }^{-}{ }^{C-P} \mathbb{D}_{p}^{\alpha_{1}(p, q)} z(p, q)+\frac{1}{4} \sin (z-v)=h_{1}(p, q), \\
{ }^{C-P} \mathbb{D}_{q}^{\alpha_{2}(p, q)} v(p, q)-{ }^{C-P} \mathbb{D}_{p}^{\alpha_{2}(p, q)} v(p, q)-\sin (z-v)=h_{2}(p, q),
\end{gathered}
$$

where

$$
\begin{aligned}
h_{1}(p, q)= & \left(q^{2-\alpha_{1}(p, q)} \mathbb{E}_{1,3-\alpha_{1}(p, q)}(-q)+e^{-q}\right) \sin (p) \\
& +\frac{1}{4} \sin \left(e^{-q}(\sin (p)-\cos (p))\right), \\
h_{2}(p, q)= & \left(q^{2-\alpha_{2}(p, q)} \mathbb{E}_{1,3-\alpha_{2}(p, q)}(-q)+e^{-q}\right) \cos (p) \\
& -\sin \left(e^{-q}(\sin (p)-\cos (p))\right),
\end{aligned}
$$

where $(p, q) \in[0,1] \times[0,1]$ and $\mathbb{E}_{\zeta, \eta}(q)=\sum_{k=0}^{\infty}\left(q^{k} / \Gamma(k \zeta+\eta)\right)$. For this example, the exact solutions to the case where $v$ $=0, \alpha_{1}(p, q)=\alpha_{2}(p, q)=2$ are $z(p, q)=\sin (p) e^{-q}, v(p, q)=$ $\cos (p) e^{-q}$. The numerical results obtained using the proposed method for $\alpha_{1}(p, q)=1.5+0.25 \sin (p q), \alpha_{2}(p, q)=$ $1.75+0.25 \sin (p q)$ are shown in Figure 1. An image of the absolute error for value $m=6$ is shown in Figure 2, and numerical results for absolute error for different values $m$ are shown in Tables 1 and 2. Table 3 shows comparison between the maximum absolute error (MAE) given for different values of $m$ based on the present and the Chebyshev cardinal function (CCF) technique [69]. In other words, the comparison between our numerical method result and the numerical method result in [69] is done using two different operators, i.e., the Caputo-

\begin{tabular}{|c|c|c|}
\hline \multirow[t]{2}{*}{$m$} & \multicolumn{2}{|c|}{$\begin{array}{c}\alpha_{1}(p, q)=1.5+0.25 \sin (p q), \alpha_{2}(p, q)=1.75 \\
+0.25 \sin (p q)\end{array}$} \\
\hline & $\operatorname{MAE}(z)$ & $\operatorname{MAE}(v)$ \\
\hline & \multicolumn{2}{|c|}{ Our method } \\
\hline 6 & $2.1048 e-16$ & $1.328 e-16$ \\
\hline 7 & $4.2097 e-18$ & $2.6561 e-17$ \\
\hline 8 & $6.3146 e-19$ & $3.9842 e-19$ \\
\hline \multirow[t]{3}{*}{9} & $6.3146 e-21$ & $3.9842 e-20$ \\
\hline & $\operatorname{MA}(u)$ & $\operatorname{MA}(w)$ \\
\hline & \multicolumn{2}{|c|}{ Second-kind CCF method [69] } \\
\hline 6 & $1.4597 e-07$ & $3.4301 e-07$ \\
\hline 7 & $2.7615 e-09$ & $1.2573 e-08$ \\
\hline 8 & $1.5434 e-10$ & $5.3747 e-10$ \\
\hline 9 & $4.0590 e-12$ & $2.0088 e-11$ \\
\hline
\end{tabular}
Prabhakar derivative and the Caputo derivative. From the obtained numerical results in Table 3, we see that the proposed method is an effective and good tool for solving this problem.
TABLE 3: Comparison of the numerical results for Example 7.

Example 8. We consider the following coupled nonlinear variable-order time-fractional sine-Gordon equations:

$$
\begin{gathered}
{ }^{C-P} \mathbb{D}_{q}^{\alpha_{1}(p, q)} z(p, q)-{ }^{C-P} \mathbb{D}_{p}^{\alpha_{1}(p, q)} z(p, q)+\sin (z-v)=h_{1}(p, q), \\
{ }^{C-P} \mathbb{D}_{q}^{\alpha_{2}(p, q)} v(p, q)-{ }^{C-P} \mathbb{D}_{p}^{\alpha_{2}(p, q)} v(p, q)-\sin (z-v)=h_{2}(p, q),
\end{gathered}
$$

where

$$
\begin{aligned}
h_{1}(p, q)= & -\left(q^{3-\alpha_{1}(p, q)} \mathbb{E}_{2,4-\alpha_{1}(p, q)}\left(-q^{2}\right)+\sin (q)\right) \cosh (p) \\
& +\sin (\sin (q)(\cosh (p)-\sinh (p))) \\
h_{2}(p, q)=- & \left(q^{3-\alpha_{2}(p, q)} \mathbb{E}_{2,4-\alpha_{2}(p, q)}\left(-q^{2}\right)+\sin (q)\right) \sinh (p) \\
& -\sin (\sin (q)(\cosh (p)-\sinh (p)))
\end{aligned}
$$




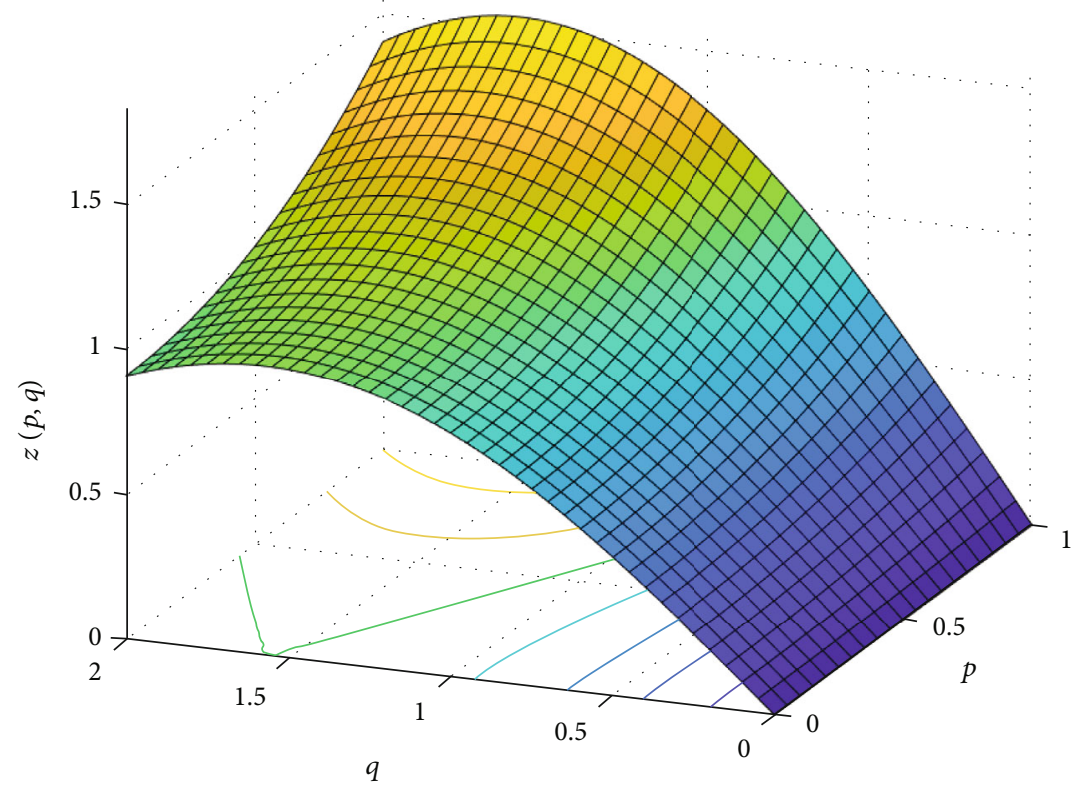

(a)

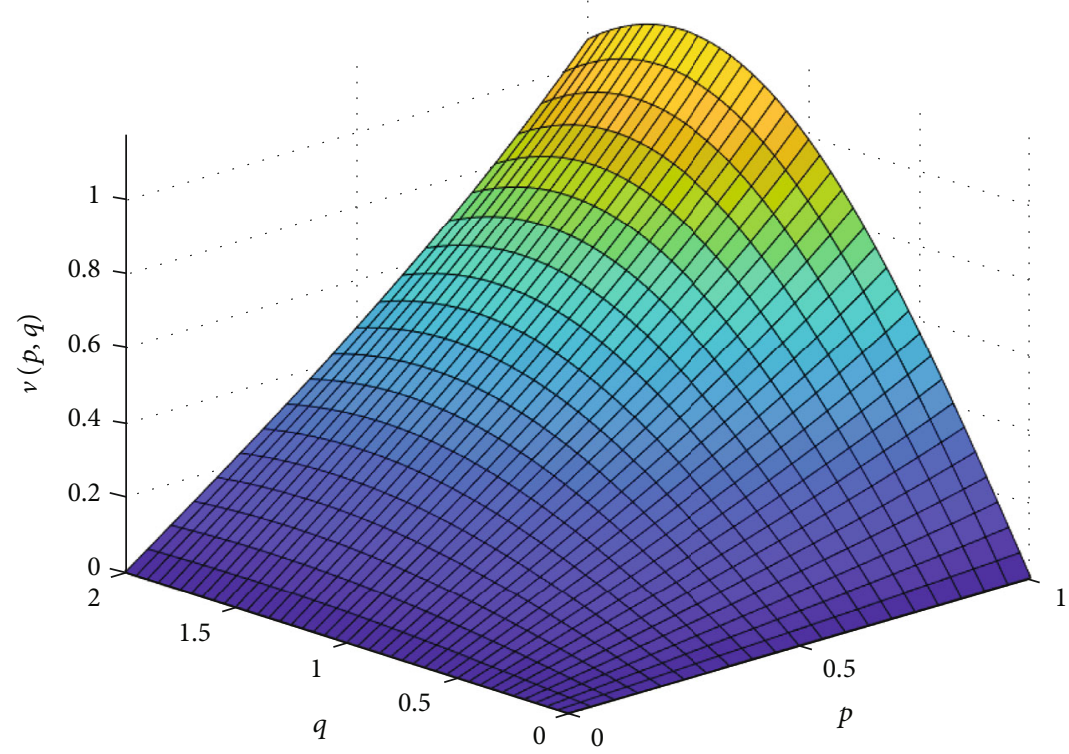

(b)

FIGURE 3: Numerical solution of $z(p, q), v(p, q)$ with $\rho=\omega=v=1$ for Example 8 when $m=6$.

where $(p, q) \in[0,1] \times[0,2]$. For this example, the exact solutions to the case where $v=0, \alpha_{1}(p, q)=\alpha_{2}(p, q)=2$ are $z(p$, $q)=\sin (q) \cosh (p), v(p, q)=\sin (q) \sinh (p)$. The numerical results obtained using the proposed method for $\alpha_{1}(p, q)$ $=1.5+0.25 p^{2} q, \alpha_{2}(p, q)=1.75+0.25 \sin (p q)$ are shown in Figure 3. An image of the absolute error for value $m=6$ is shown in Figure 4, and numerical results for the absolute error for different values $m$ are shown in Tables 4 and 5 . The values of the MAE, $C$-order, and CPU time of the presented method are listed in Table 6.
Example 9. We consider the following coupled nonlinear variable-order time-fractional sine-Gordon equations:

$$
\begin{aligned}
& { }^{C-P} \mathbb{D}_{q}^{\alpha_{1}(p, q)} z(p, q)-{ }^{C-P} \mathbb{D}_{p}^{\alpha_{1}(p, q)} z(p, q)+\sin (z-v)=h_{1}(p, q), \\
& { }^{C-P} \mathbb{D}_{q}^{\alpha_{2}(p, q)} v(p, q)-\frac{1}{4}{ }^{C-P} \mathbb{D}_{p}^{\alpha_{2}(p, q)} v(p, q)-\sin (z-v)=h_{2}(p, q),
\end{aligned}
$$




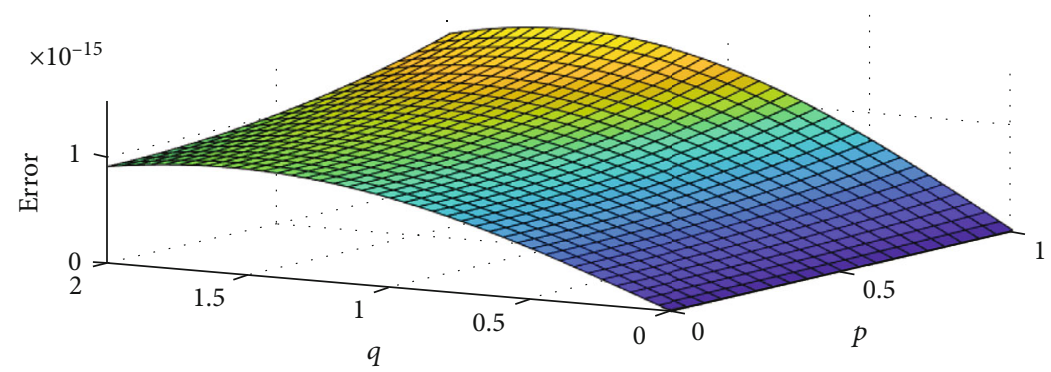

(a)

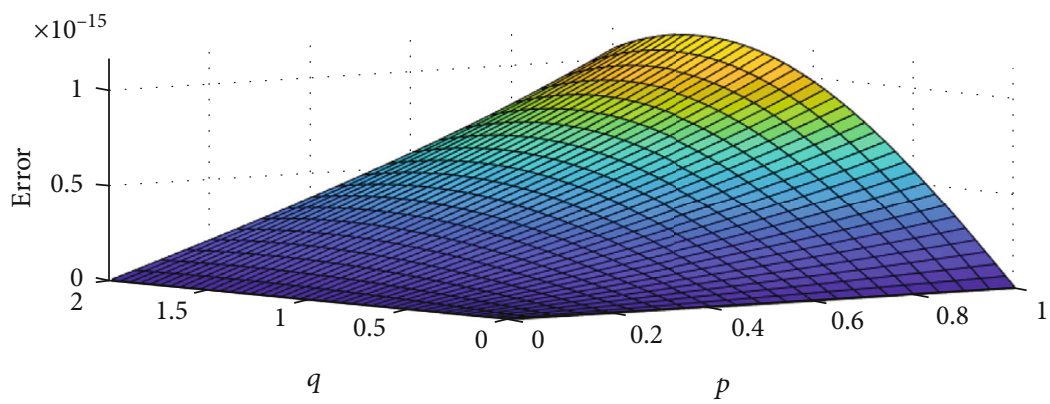

(b)

Figure 4: Charts of absolute error $z$ (a) and $v(\mathrm{~b})$ with $\rho=\omega=v=1$ for Example 8 when $m=6$.

TABle 4: The absolute error for different values $m$ for Example 8 at various points $(p, q)$.

\begin{tabular}{lcccc}
\hline$(p, q)$ & $m=6$ & $m=7$ & $m=8$ & $m=9$ \\
\hline$(0.2,0.2)$ & $1.9744 e-16$ & $1.3821 e-15$ & $1.5795 e-15$ & $1.7770 e-15$ \\
$(0.4,0.4)$ & $3.9281 e-16$ & $2.7497 e-15$ & $3.1425 e-15$ & $3.5353 e-15$ \\
$(0.6,0.6)$ & $5.8368 e-16$ & $4.0857 e-15$ & $4.6694 e-15$ & $5.2531 e-15$ \\
$(0.8,0.8)$ & $7.6689 e-16$ & $5.3682 e-15$ & $6.1351 e-15$ & $6.9020 e-15$ \\
$(1,1)$ & $9.3832 e-16$ & $6.5682 e-15$ & $7.5065 e-15$ & $8.4449 e-15$ \\
$(0.2,1.2)$ & $1.0926 e-15$ & $7.6483 e-15$ & $8.7409 e-15$ & $9.8336 e-15$ \\
$(0.4,1.4)$ & $1.2231 e-15$ & $8.5621 e-15$ & $9.7853 e-15$ & $1.1008 e-14$ \\
$(0.6,1.6)$ & $1.3220 e-15$ & $9.2540 e-15$ & $1.0576 e-14$ & $1.1898 e-14$ \\
$(0.8,1.8)$ & $1.3801 e-15$ & $9.6607 e-15$ & $1.1040 e-14$ & $1.2420 e-14$ \\
$(1,2)$ & $1.3875 e-15$ & $9.7127 e-15$ & $1.1100 e-14$ & $1.2487 e-14$ \\
\hline
\end{tabular}

where

$$
\begin{aligned}
& h_{1}(p, q)=p^{3}(p-2) q^{2-\alpha_{1}(p, q)} \mathbb{E}_{2,3-\alpha_{1}(p, q)}\left(-q^{2}\right)+6 p(2 p-2) \cos (q) \\
&+\sin \left(p^{3}(2-p)(\cos (q)-\sin (q))\right) \\
& h_{2}(p, q)= p^{3}(p-2) q^{3-\alpha_{2}(p, q)} \mathbb{E}_{2,4-\alpha_{1}(p, q)}\left(-q^{2}\right) \\
&+\frac{3}{2} p(2 p-2) \sin (q) \\
&-\sin \left(p^{3}(2-p)(\cos (q)-\sin (q))\right)
\end{aligned}
$$

TABLE 5: The absolute error for different values $m$ for Example 8 at various points $(p, q)$.

\begin{tabular}{lcccc}
\hline \multirow{2}{*}{$p, q)$} & \multicolumn{4}{c}{$\left|v_{m}-v\right|$} \\
& $m=6$ & $m=7$ & $m=8$ & $m=9$ \\
\hline$(0.2,0.2)$ & $1.9678 e-17$ & $1.3775 e-16$ & $1.5743 e-16$ & $1.7711 e-16$ \\
$(0.4,0.4)$ & $7.7532 e-17$ & $5.4272 e-16$ & $6.2026 e-16$ & $6.9779 e-16$ \\
$(0.6,0.6)$ & $1.7003 e-16$ & $1.1902 e-15$ & $1.3602 e-15$ & $1.5303 e-15$ \\
$(0.8,0.8)$ & $2.9138 e-16$ & $2.0396 e-15$ & $2.3310 e-15$ & $2.6224 e-15$ \\
$(1,1)$ & $4.3361 e-16$ & $3.0353 e-15$ & $3.4689 e-15$ & $3.9025 e-15$ \\
$(0.2,1.2)$ & $5.8679 e-16$ & $4.1075 e-15$ & $4.6943 e-15$ & $5.2811 e-15$ \\
$(0.4,1.4)$ & $7.3924 e-16$ & $5.1746 e-15$ & $5.9139 e-15$ & $6.6531 e-15$ \\
$(0.6,1.6)$ & $8.7786 e-16$ & $6.1450 e-15$ & $7.0229 e-15$ & $7.9007 e-15$ \\
$(0.8,1.8)$ & $9.8856 e-16$ & $6.9199 e-15$ & $7.9085 e-15$ & $8.8970 e-15$ \\
$(1,2)$ & $1.0567 e-15$ & $7.3971 e-15$ & $8.4538 e-15$ & $9.5106 e-15$ \\
\hline
\end{tabular}

TABle 6: The MA-error, $C$-order, and CPU time of the presented method in Example 8.

\begin{tabular}{cccccc}
\hline \multirow{2}{*}{$z(p, q)$} & \multicolumn{2}{c}{$v(p, q)$} & CPU-time $(\mathrm{s})$ \\
& MAE & $C$-order & MAE & $C$-order & \\
\hline 6 & $1.1801 e-15$ & - & $1.0547 e-15$ & - & 6.15 \\
7 & $7.5127 e-16$ & 5.7033 & $7.3971 e-16$ & 07.6726 & 18.14 \\
8 & $1.0100 e-16$ & 15.0260 & $6.4538 e-16$ & 11.3146 & 44.13 \\
9 & $1.0487 e-17$ & 16.9570 & $9.5106 e-17$ & 11.4025 & 87.14 \\
\hline
\end{tabular}




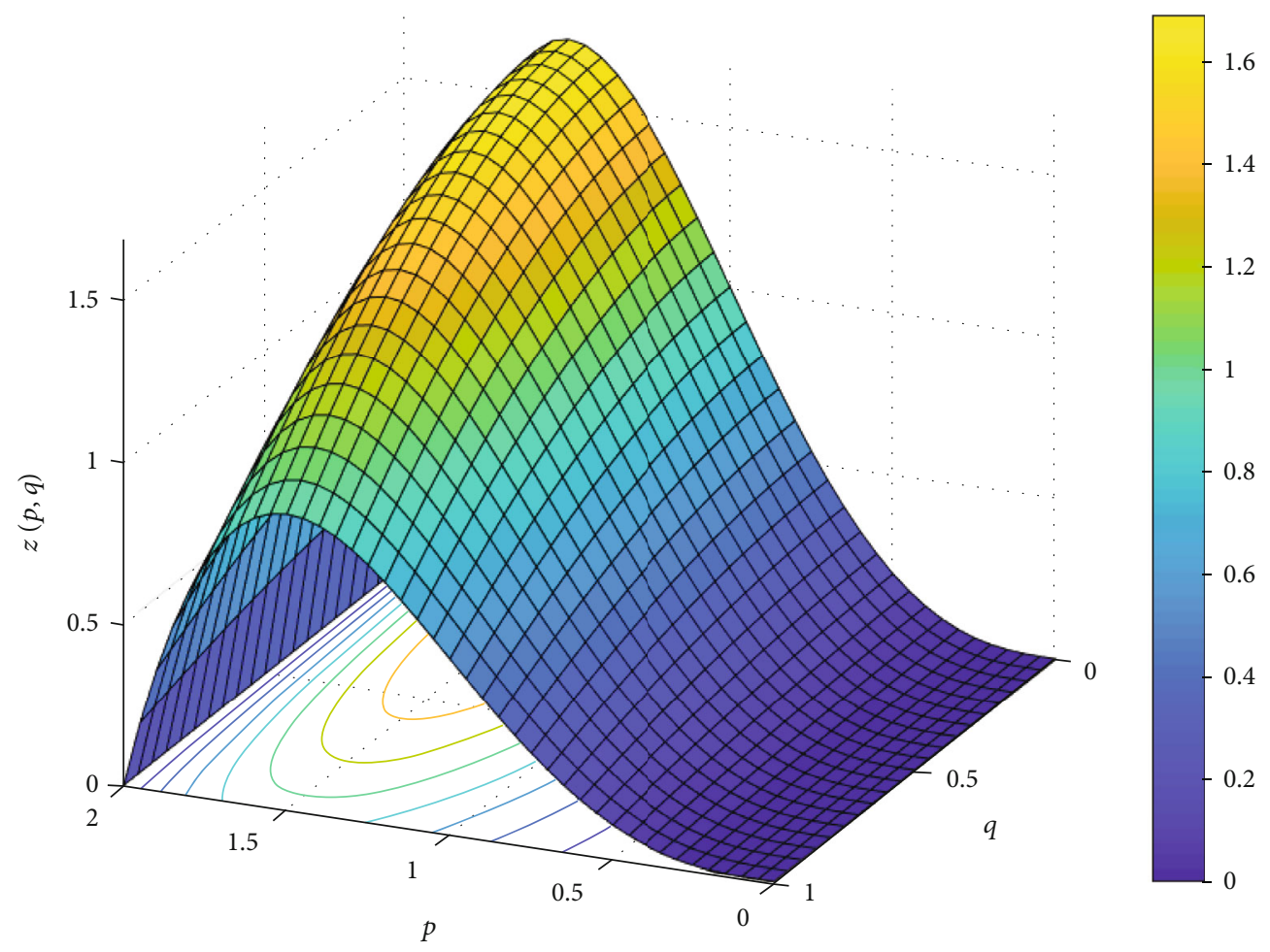

(a)

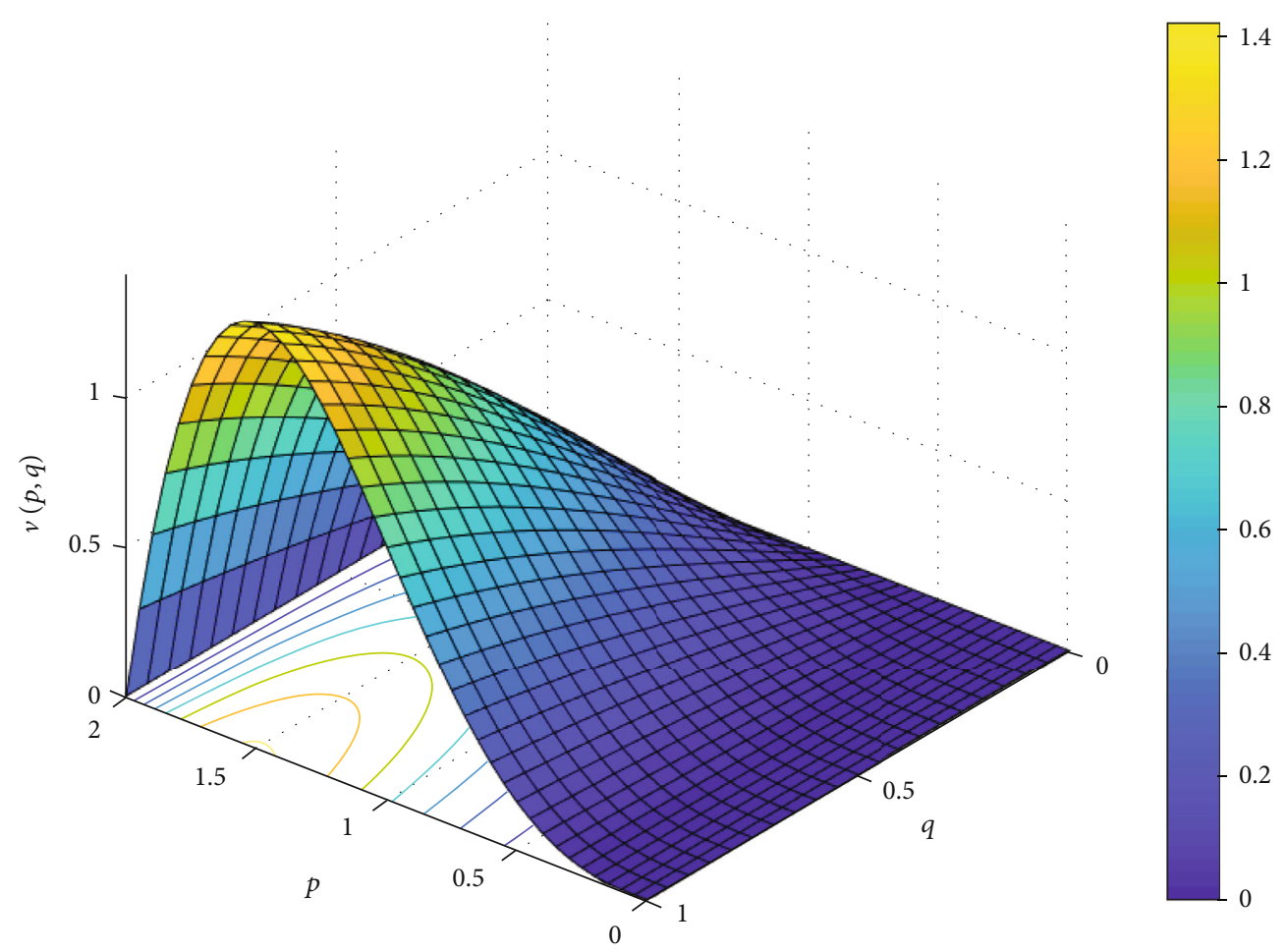

(b)

FIgURE 5: Numerical solution of $z(p, q), v(p, q)$ with $\rho=\omega=v=1$ for Example 9 when $m=6$. 


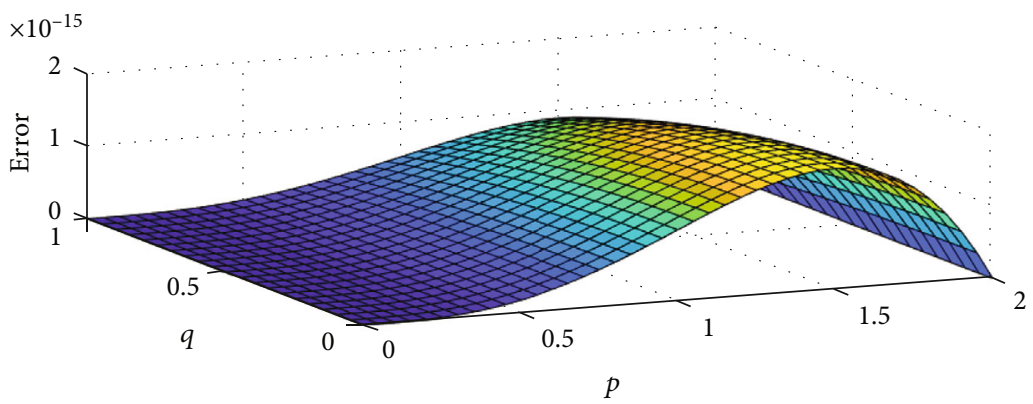

(a)

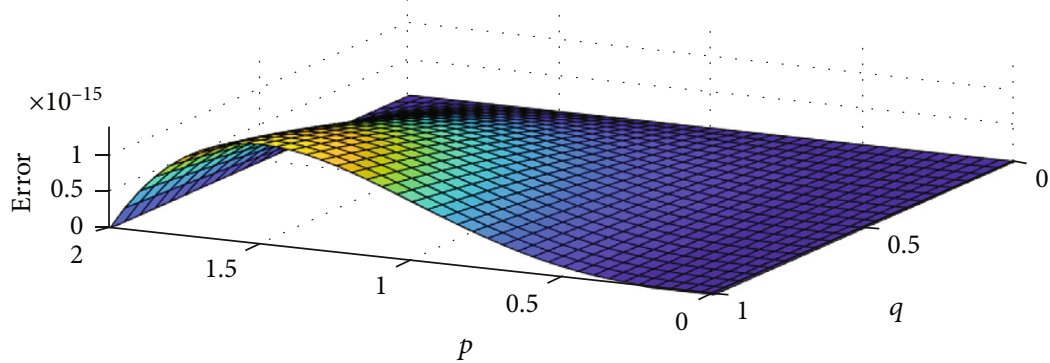

(b)

Figure 6: Charts of absolute error $z$ (a) and $v$ (b) with $\rho=\omega=v=1$ for Example 9 when $m=6$.

TABle 7: The absolute error for different values $m$ for Example 9 at various points $(p, q)$.

\begin{tabular}{lcccc}
\hline$(p, q)$ & \multicolumn{4}{c}{$\left|z_{m}-z\right|$} \\
& $m=6$ & $m=7$ & $m=8$ & $m=9$ \\
\hline$(0.2,0.2)$ & $1.4168 e-17$ & $9.9182 e-17$ & $1.1335 e-16$ & $1.2751 e-16$ \\
$(0.4,0.4)$ & $9.9243 e-17$ & $6.9470 e-16$ & $7.9395 e-16$ & $8.9319 e-16$ \\
$(0.6,0.6)$ & $2.8568 e-16$ & $1.9997 e-15$ & $2.2854 e-15$ & $2.5711 e-15$ \\
$(0.8,0.8)$ & $5.5961 e-16$ & $3.9172 e-15$ & $4.4769 e-15$ & $5.0365 e-15$ \\
$(1,1)$ & $8.6783 e-16$ & $6.0748 e-15$ & $6.9426 e-15$ & $7.8104 e-15$ \\
$(1.2,0.2)$ & $1.1282 e-15$ & $7.8978 e-15$ & $9.0261 e-15$ & $1.0154 e-14$ \\
$(1.4,0.4)$ & $1.2452 e-15$ & $8.7167 e-15$ & $9.9619 e-15$ & $1.1207 e-14$ \\
$(1.6,0.6)$ & $1.1288 e-15$ & $7.9016 e-15$ & $9.0304 e-15$ & $1.0159 e-14$ \\
$(1.8,0.8)$ & $7.1699 e-16$ & $5.0189 e-15$ & $5.7359 e-15$ & $6.4529 e-15$ \\
\hline
\end{tabular}

TABle 8: The absolute error for different values $m$ for Example 9 at various points $(p, q)$.

\begin{tabular}{lcccc}
\hline \multirow{2}{*}{$p, q)$} & \multicolumn{4}{c}{$\left|v_{m}-v\right|$} \\
& $m=6$ & $m=7$ & $m=8$ & $m=9$ \\
\hline$(0.2,0.2)$ & $1.4216 e-18$ & $9.9514 e-18$ & $1.1373 e-17$ & $1.2794 e-17$ \\
$(0.4,0.4)$ & $2.0117 e-17$ & $1.4082 e-16$ & $1.6094 e-16$ & $1.8105 e-16$ \\
$(0.6,0.6)$ & $8.8372 e-17$ & $6.1860 e-16$ & $7.0697 e-16$ & $7.9535 e-16$ \\
$(0.8,0.8)$ & $2.3660 e-16$ & $1.6562 e-15$ & $1.8928 e-15$ & $2.1294 e-15$ \\
$(1,1)$ & $4.7409 e-16$ & $3.3186 e-15$ & $3.7927 e-15$ & $4.2668 e-15$ \\
$(1.2,0.2)$ & $7.7188 e-16$ & $5.4032 e-15$ & $6.1751 e-15$ & $6.9470 e-15$ \\
$(1.4,0.4)$ & $1.0488 e-15$ & $7.3419 e-15$ & $8.3908 e-15$ & $9.4397 e-15$ \\
$(1.6,0.6)$ & $1.1622 e-15$ & $8.1358 e-15$ & $9.2980 e-15$ & $1.0460 e-14$ \\
$(1.8,0.8)$ & $9.0352 e-16$ & $6.3246 e-15$ & $7.2281 e-15$ & $8.1316 e-15$ \\
\hline
\end{tabular}

TABle 9: The MAE, $C$-order, and CPU time of the presented method in Example 9.

\begin{tabular}{cccccc}
\hline \multirow{2}{*}{$\begin{array}{c}c \\
m\end{array}$} & MAE & $C$-order & MAE & $C$-order & \\
\hline 6 & $7.2352 e-15$ & - & $1.1522 e-15$ & - & 8.17 \\
7 & $4.7067 e-15$ & 2.7023 & $8.1258 e-16$ & 6.6706 & 20.04 \\
8 & $1.9519 e-16$ & 12.0250 & $7.2880 e-16$ & 10.3156 & 46.10 \\
9 & $6.8004 e-17$ & 13.9560 & $9.4297 e-17$ & 10.4015 & 89.04 \\
\hline
\end{tabular}

where $(p, q) \in[0,2] \times[0,1]$. For this example, the exact solutions to the case where $v=0, \alpha_{1}(p, q)=\alpha_{2}(p, q)=2$ are $z(p, q)=p^{3}(2-p) \cos (q), v(p, q)=p^{3}(2-p) \sin (q)$. The numerical results obtained using the proposed method for $\alpha_{1}(p, q)=1.5+0.25 \sin (p q), \alpha_{2}(p, q)=1.75+0.25 \sin (p q)$ are shown in Figure 5. An image of the absolute error for value $m=6$ is shown in Figure 6, and numerical results for absolute error for different values $m$ are shown in Tables 7 and 8 . The values of the MAE, $C$-order, and CPU time of the presented method are listed in Table 9.

Example 10. We consider the following coupled nonlinear variable-order time-fractional sine-Gordon equations:

$$
\begin{aligned}
& { }^{C-P} \mathbb{D}_{q}^{\alpha_{1}(p, q)} z(p, q)-{ }^{C-P} \mathbb{D}_{p}^{\alpha_{1}(p, q)} z(p, q)+4 \sin (z-v)=h_{1}(p, q), \\
& { }^{C-P} \mathbb{D}_{q}^{\alpha_{2}(p, q)} v(p, q)-9^{C-P} \mathbb{D}_{p}^{\alpha_{2}(p, q)} v(p, q)-\sin (z-v)=h_{2}(p, q),
\end{aligned}
$$




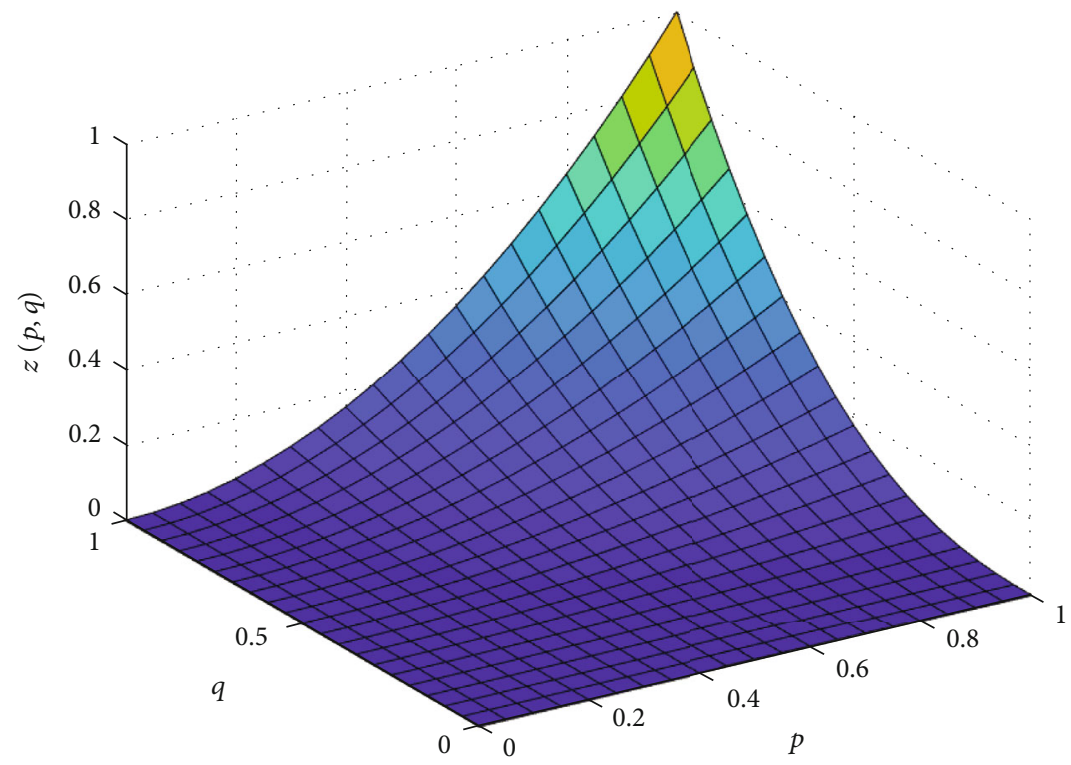

(a)

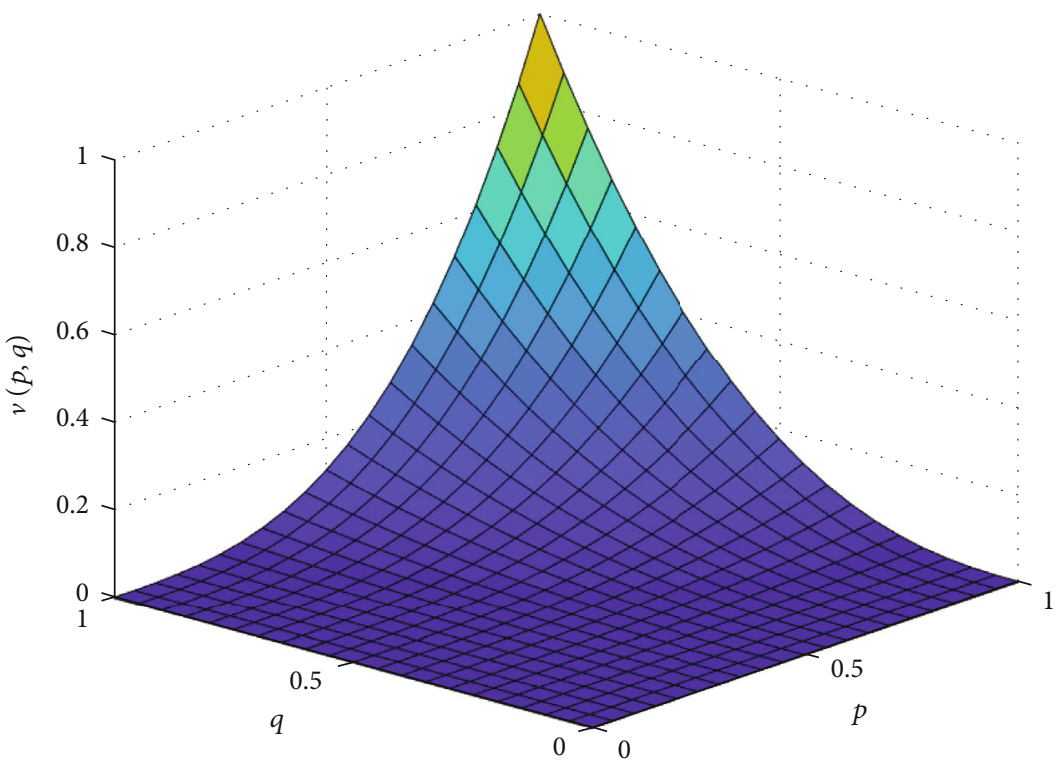

(b)

Figure 7: Numerical solution of $z(p, q), v(p, q)$ with $\rho=\omega=v=1$ for Example 10 when $m=6$.

where

$$
\begin{aligned}
h_{1}(p, q)= & \frac{\Gamma(7 / 2)}{\Gamma(7 / 2)-\alpha_{1}(p, q)} p^{2} q^{5 / 2-\alpha_{1}(p, q)}-2 q^{5 / 2} \\
& +4 \sin \left(\left(p^{2}-p^{3}\right) q^{5 / 2}\right), \\
h_{2}(p, q)= & \frac{\Gamma(7 / 2)}{\Gamma(7 / 2)-\alpha_{2}(p, q)} p^{3} q^{5 / 2-\alpha_{1}(p, q)}-54 p q^{5 / 2} \\
& -\sin \left(\left(p^{2}-p^{3}\right) q^{5 / 2}\right),
\end{aligned}
$$

where $(p, q) \in[0,1] \times[0,1]$. For this example, the exact solutions to the case where $v=0, \alpha_{1}(p, q)=\alpha_{2}(p, q)=2$ are $z(p, q)=p^{2} q^{5 / 2}, v(p, q)=p^{3} q^{5 / 2}$. The numerical results obtained using the proposed method for $\alpha_{1}(p, q)=1.65+$ $0.25 \sin (p q), \alpha_{2}(p, q)=1.55+0.35 \sin (p q)$ are shown in Figure 7. An image of the absolute error for value $m=6$ is shown in Figure 8, and numerical results for absolute error for different values $m$ are shown in Tables 10-12. Table 12 shows the comparison between the maximum absolute error (MAE) given for different values of $m$ based on the present and the Chebyshev cardinal function (CCF) 


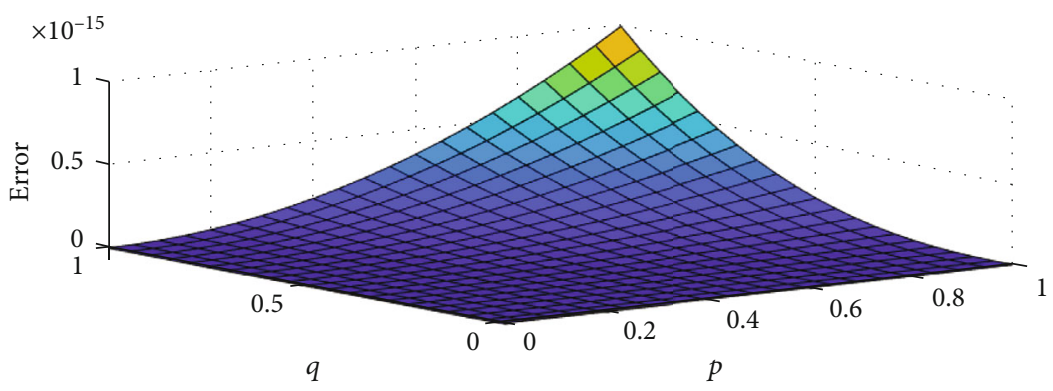

(a)

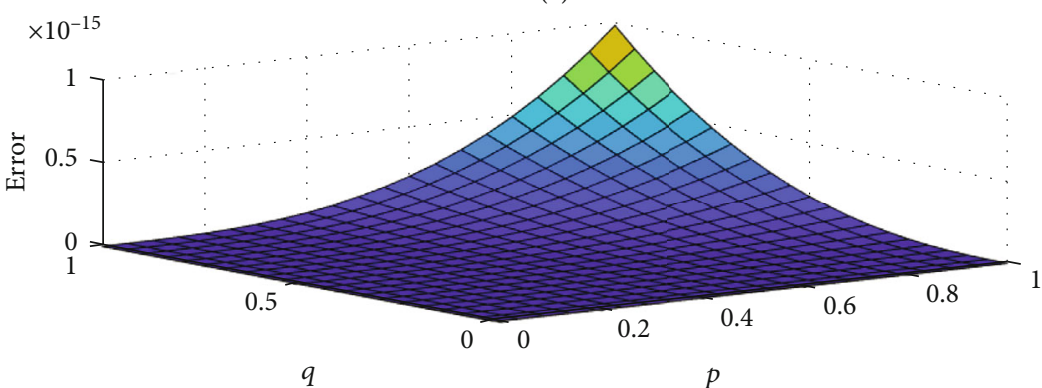

(b)

Figure 8: Charts of absolute error $z$ (a) and $v$ (b) with $\rho=\omega=v=1$ for Example 10 when $m=6$.

TABLE 10: The absolute error for different values $m$ for Example 10 at various points $(p, q)$.

\begin{tabular}{|c|c|c|c|c|}
\hline \multirow{2}{*}{$(p, q)$} & \multicolumn{4}{|c|}{$\left|z_{m}-z\right|$} \\
\hline & $m=6$ & $m=7$ & $m=8$ & $m=9$ \\
\hline$(0.1,0.1)$ & $3.1271 e-20$ & $2.1890 e-19$ & $2.50179 e-19$ & $2.8144 e-19$ \\
\hline$(0.3,0.3)$ & $4.3872 e-18$ & $3.0710 e-17$ & $3.5098 e-17$ & $3.9485 e-17$ \\
\hline$(0.5,0.5)$ & $4.3703 e-17$ & $3.0592 e-16$ & $3.4962 e-16$ & $3.9332 e-16$ \\
\hline$(0.7,0.7)$ & $1.9865 e-16$ & $1.3905 e-15$ & $1.5892 e-15$ & $1.7878 e-15$ \\
\hline$(0.9,0.9)$ & $6.1551 e-16$ & $4.3086 e-15$ & $4.9241 e-15$ & $5.5396 e-15$ \\
\hline
\end{tabular}

TABle 11: The absolute error for different values $m$ for Example 10 at various points $(p, q)$.

\begin{tabular}{lcccc}
\hline \multirow{2}{*}{$(p, q)$} & $m=6$ & $m=7$ & $m=8$ & $m=9$ \\
\hline$(0.1,0.1)$ & $3.1271 e-21$ & $2.1890 e-20$ & $2.5017 e-20$ & $2.8144 e-20$ \\
$(0.3,0.3)$ & $1.3161 e-18$ & $9.2132 e-18$ & $1.0529 e-17$ & $1.1845 e-17$ \\
$(0.5,0.5)$ & $2.1851 e-17$ & $1.5296 e-16$ & $1.7481 e-16$ & $1.9666 e-16$ \\
$(0.7,0.7)$ & $1.3905 e-16$ & $9.7338 e-16$ & $1.1124 e-15$ & $1.2514 e-15$ \\
$(0.9,0.9)$ & $5.5396 e-16$ & $3.8777 e-15$ & $4.4317 e-15$ & $4.9856 e-15$ \\
\hline
\end{tabular}

technique [69]. In other words, the comparison between our numerical method result and the numerical method result in [69] is done with using two different operators, i.e., the Caputo-Prabhakar derivative and the Caputo derivative.
TABle 12: Comparison of the numerical results for Example 10.

\begin{tabular}{lcc}
\hline \multicolumn{4}{c}{$\alpha_{1}(p, q)=1.65+0.25 \sin (p q), \alpha_{2}(p, q)=$} \\
$m$ & $\operatorname{MAE}(z)$ & Our method \\
& \multicolumn{3}{c}{$\operatorname{MAE}(v)$} \\
\hline 6 & $3.1131 e-18$ & $2.1440 e-19$ \\
7 & $1.3161 e-18$ & $7.2122 e-20$ \\
8 & $2.1941 e-19$ & $1.4596 e-20$ \\
9 & $1.2605 e-19$ & $8.5238 e-21$ \\
& $\operatorname{MAE}(u)$ & $\operatorname{MAE}(w)$ \\
& Second-kind CCF method $[69]$ \\
6 & $6.5157 e-04$ & $1.7826 e-05$ \\
7 & $4.1287 e-04$ & $9.8284 e-06$ \\
8 & $2.7169 e-04$ & $5.8208 e-06$ \\
9 & $2.0393 e-04$ & $2.9727 e-06$ \\
\hline
\end{tabular}




\section{Conclusion}

This paper is focused on a numerical method based on the orthogonal polynomials known as the shifted Chebyshev polynomials of degreem. The fractional sine-Gordon equations of variable order which includes a fractional derivative in terms of the Caputo-Prabhakar sense in this paper are studied. Using this numerical method, we obtain the numerical solutions of this type of equations. In this article, the convergence and error analysis for numerical solutions are studied. Some numerical examples are shown for the application of numerical methods.

\section{Data Availability}

No data were used to support the study.

\section{Conflicts of Interest}

The author declares that he has no conflict of interest.

\section{References}

[1] K. S. Miller and B. Ross, An Introduction to The Fractional Calculus and Fractional Differential Equations, John-Wiley and Sons. Inc., New York, 1993.

[2] I. Podlubny, Fractional differential equations, vol. 198 of Mathematics in Science and Engineering, Academic Press, 1999.

[3] S. G. Samko, A. A. Kilbas, and O. I. Marichev, Fractional Integrals and Derivatives (Vol. 1), Gordon and Breach Science Publishers, Yverdon-les-Bains, Switzerland, 1993.

[4] C. F. Coimbra, "Mechanics with variable-order differential operators," Annalen der Physik, vol. 12, no. 1112, pp. 692703, 2003.

[5] K. Diethelm and N. J. Ford, "Analysis of fractional differential equations," Journal of Mathematical Analysis and Applications, vol. 265, no. 2, pp. 229-248, 2002.

[6] F. Mainardi, "The fundamental solutions for the fractional diffusion-wave equation," Applied Mathematics Letters, vol. 9, no. 6, pp. 23-28, 1996.

[7] H. T. C. Pedro, M. H. Kobayashi, J. M. C. Pereira, and C. F. M. Coimbra, "Variable order modeling of diffusive-convective effects on the oscillatory flow past a sphere," Journal of Vibration and Control, vol. 14, no. 9-10, pp. 1659-1672, 2008.

[8] L. E. S. Ramirez and C. F. M. Coimbra, "On the variable order dynamics of the nonlinear wake caused by a sedimenting particle," Physica D: Nonlinear Phenomena, vol. 240, no. 13, pp. 1111-1118, 2011.

[9] K. B. Oldham, "Fractional differential equations in electrochemistry," Advances in Engineering Software, vol. 41, no. 1, pp. 9-12, 2010.

[10] Y. Povstenko, "Generalized theory of diffusive stresses associated with the time-fractional diffusion equation and nonlocal constitutive equations for the stress tensor," Computers \& Mathematics with Applications, vol. 78, no. 6, pp. 1819-1825, 2019.

[11] W.-P. Zhong and M. Belić, "Special two-soliton solution of the generalized Sine-Gordon equation with a variable coefficient," Applied Mathematics Letters, vol. 38, pp. 122-128, 2014.
[12] K. R. Khusnutdinova and D. E. Pelinovsky, "On the exchange of energy in coupled Klein-Gordon equations," Wave Motion, vol. 38, no. 1, pp. 1-10, 2003.

[13] O. M. Braun and Y. S. Kivshar, "Nonlinear dynamics of the Frenkel-Kontorova model," Physics Reports, vol. 306, no. 1-2, pp. 1-108, 1998.

[14] T. Kontorova and J. Frenkel, "On the theory of plastic deformation and twinning. II," Zhurnal Eksperimental'noi i Teoreticheskoi Fiziki, vol. 8, pp. 1340-1348, 1938.

[15] S. Yomosa, "Soliton excitations in deoxyribonucleic acid (DNA) double helices," Physical Review A, vol. 27, no. 4, pp. 2120-2125, 1983.

[16] R. Garra, R. Gorenflo, F. Polito, and Ž. Tomovski, "HilferPrabhakar derivatives and some applications," Applied Mathematics and Computation, vol. 242, pp. 576-589, 2014.

[17] T. R. Prabhakar, "A singular integral equation with a generalized Mittag Leffler function in the kernel," Yokohama Mathematical Journal, vol. 19, pp. 7-15, 1971.

[18] F. Alvarez, A. Alegra, and J. Colmenero, "Relationship between the time-domain Kohlrausch-Williams-Watts and frequencydomain Havriliak-Negami relaxation functions," Physical Review B, vol. 44, no. 14, pp. 7306-7312, 1991.

[19] P. Miskinis, “The Havriliak-Negami susceptibility as a nonlinear and nonlocal process.," Physica Scripta, vol. T136, article 014019, 2009.

[20] A. Stanislavsky and K. Weron, "Atypical case of the dielectric relaxation responses and its fractional kinetic equation," Fractional Calculus and Applied Analysis, vol. 19, no. 1, pp. 212228, 2016.

[21] S. C. Pandey, “The Lorenzo-Hartley's function for fractional calculus and its applications pertaining to fractional order modelling of anomalous relaxation in dielectrics," Computational and Applied Mathematics, vol. 37, no. 3, pp. 26482666, 2018.

[22] M. D'Ovidio and F. Polito, "Fractional diffusion-telegraph equations and their associated stochastic solutions," Theory of Probability \& Its Applications, vol. 62, no. 4, pp. 552-574, 2018.

[23] R. Hilfer, "Fractional calculus and regular variation in thermodynamics," in Applications of Fractional Calculus in Physics, R. Hilfer, Ed., pp. 429-463, World Scientific, Singapore, 2000.

[24] R. Hilfer and L. Anton, "Fractional master equations and fractal time random walks," Physical Review E, vol. 51, no. 2, p. R848, 1995.

[25] Y. Hu and G. Wang, "Weak solutions to a nonlinear variational sine-Gordon equation," Journal of Mathematical Analysis and Applications, vol. 402, no. 1, pp. 1-11, 2013.

[26] X. Li, S. Zhang, Y. Wang, and H. Chen, "Analysis and application of the element-free Galerkin method for nonlinear sineGordon and generalized sinh-Gordon equations," Computers \& Mathematics with Applications, vol. 71, no. 8, pp. 16551678, 2016.

[27] E. H. Doha, M. A. Abdelkawy, A. Z. M. Amin, and A. M. Lopes, "A space-time spectral approximation for solving nonlinear variable-order fractional sine and Klein-Gordon differential equations," Computational and Applied Mathematics, vol. 37, no. 5, pp. 6212-6229, 2018.

[28] S. C. Lim and C. H. Eab, "Fractional quantum fields," in Applications in Physics, Part B, V. E. Tarasov, Ed., pp. 237-256, De Gruyter, 2019. 
[29] D. Lu and S. Ye, "Optical solitary wave solutions of the space-time fractional modified equal-width equation and their applications," International Journal, vol. 8, no. 1, pp. 1-20, 2019.

[30] S. Guo, L. Mei, Y. Hou, and Z. Zhang, "An efficient finite difference/Hermite-Galerkin spectral method for time- fractional coupled sine-Gordon equations on multidimensional unbounded domains and its application in numerical simulations of vector solitons," Computer Physics Communications, vol. 237, pp. 110-128, 2019.

[31] M. Ilati and M. Dehghan, "The use of radial basis functions (RBFs) collocation and RBF-QR methods for solving the coupled nonlinear sine-Gordon equations," Engineering Analysis with Boundary Elements, vol. 52, pp. 99-109, 2015.

[32] A. Ahmadian, S. Salahshour, M. Ali-Akbari, F. Ismail, and D. Baleanu, "A novel approach to approximate fractional derivative with uncertain conditions," Chaos, Solitons \& Fractals, vol. 104, pp. 68-76, 2017.

[33] A. Ahmadian, F. Ismail, S. Salahshour, D. Baleanu, and F. Ghaemi, "Uncertain viscoelastic models with fractional order: a new spectral tau method to study the numerical simulations of the solution," Communications in Nonlinear Science and Numerical Simulation, vol. 53, pp. 44-64, 2017.

[34] Y. Chen, L. Liu, B. Li, and Y. Sun, "Numerical solution for the variable order linear cable equation with Bernstein polynomials," Applied Mathematics and Computation, vol. 238, pp. 329-341, 2014.

[35] M. Dehghan, M. Abbaszadeh, and A. Mohebbi, “An implicit RBF meshless approach for solving the time fractional nonlinear sine-Gordon and Klein-Gordon equations," Engineering Analysis with Boundary Elements, vol. 50, pp. 412-434, 2015.

[36] E. H. Doha, M. A. Abdelkawy, A. Z. M. Amin, and D. Baleanu, "Spectral technique for solving variable-order fractional Volterra integro-differential equations," Numerical Methods for Partial Differential Equations, vol. 34, no. 5, pp. 1659-1677, 2018.

[37] M. Kurulay, "Solving the fractional nonlinear Klein-Gordon equation by means of the homotopy analysis method," Advances in Difference Equations, vol. 2012, no. 1, pp. 1-8, 2012.

[38] Y. Gu and H. Sun, "A meshless method for solving threedimensional time fractional diffusion equation with variableorder derivatives," Applied Mathematical Modelling, vol. 78, pp. 539-549, 2020.

[39] M. Lakestani and M. Dehghan, "Collocation and finite difference-collocation methods for the solution of nonlinear Klein-Gordon equation," Computer Physics Communications, vol. 181, no. 8, pp. 1392-1401, 2010.

[40] A. Kanwal, C. Phang, and U. Iqbal, "Numerical solution of fractional diffusion wave equation and fractional Klein-Gordon equation via two-dimensional Genocchi polynomials with a Ritz-Galerkin method," Computation, vol. 6, no. 3, p. 40, 2018.

[41] A. Kanwal, C. Phang, and J. Loh, "New collocation scheme for solving fractional partial differential equations," Hacettepe Journal of Mathematics and Statistics, vol. 49, no. 3, pp. 1107-1125, 2020.

[42] A. M. Nagy, "Numerical solution of time fractional nonlinear Klein-Gordon equation using Sinc-Chebyshev collocation method," Applied Mathematics and Computation, vol. 310, pp. 139-148, 2017.
[43] A. H. Bhrawy and M. A. Zaky, "Numerical algorithm for the variable-order Caputo fractional functional differential equation," Nonlinear Dynamics, vol. 85, no. 3, pp. 1815-1823, 2016.

[44] R. Dehghan, "A numerical solution of variable order fractional functional differential equation based on the shifted Legendre polynomials," SeMA Journal, vol. 76, no. 2, pp. 217-226, 2019.

[45] F. K. Keshi, B. P. Moghaddam, and A. Aghili, "A numerical technique for variable-order fractional functional nonlinear dynamic systems," International Journal of Dynamics and Control, vol. 7, no. 4, pp. 1350-1357, 2019.

[46] M. H. Heydari and Z. Avazzadeh, "A new wavelet method for variable-order fractional optimal control problems," Asian Journal of Control, vol. 20, no. 5, pp. 1804-1817, 2018.

[47] M. H. Heydari and Z. Avazzadeh, "Legendre wavelets optimization method for variable-order fractional Poisson equation," Chaos, Solitons \& Fractals, vol. 112, pp. 180-190, 2018.

[48] A. S. Moghadam, M. Arabameri, D. Baleanu, and M. Barfeie, "Numerical solution of variable fractional order advectiondispersion equation using Bernoulli wavelet method and new operational matrix of fractional order derivative," Mathematical Methods in the Applied Sciences, vol. 43, no. 7, pp. 39363953, 2020.

[49] F. Mohammadi and H. Hassani, "Numerical solution of twodimensional variable-order fractional optimal control problem by generalized polynomial basis," Journal of Optimization Theory and Applications, vol. 180, no. 2, pp. 536-555, 2019.

[50] J. Alidousti, "Stability region of fractional differential systems with Prabhakar derivative," Journal of Applied Mathematics and Computing, vol. 62, no. 1-2, pp. 135-155, 2020.

[51] M. Ahmadinia and Z. Safari, "Analysis of local discontinuous Galerkin method for time-space fractional sine-Gordon equations," Applied Numerical Mathematics, vol. 148, pp. 1-17, 2020.

[52] M. Ahmadinia, Z. Safari, and S. Fouladi, "Analysis of local discontinuous Galerkin method for time-space fractional convection-diffusion equations," BIT Numerical Mathematics, vol. 58, no. 3, pp. 533-554, 2018.

[53] A. S. Hendy and M. A. Zaky, "Graded mesh discretization for coupled system of nonlinear multi-term time-space fractional diffusion equations," Engineering with Computers, 2020.

[54] M. A. Zaky, A. S. Hendy, and J. E. Macías-Díaz, "High-order finite difference/spectral-Galerkin approximations for the nonlinear time-space fractional Ginzburg-Landau equation," Numerical Methods for Partial Differential Equations, 2020.

[55] A. H. Bhrawy and M. Zaky, "An improved collocation method for multi-dimensional space-time variable-order fractional Schrodinger equations," Applied Numerical Mathematics, vol. 111, pp. 197-218, 2017.

[56] A. H. Bhrawy and M. A. Zaky, "Highly accurate numerical schemes for multi-dimensional space variable-order fractional Schrodinger equations," Computers \& Mathematics with Applications, vol. 73, no. 6, pp. 1100-1117, 2017.

[57] A. Mahmoud, I. G. Ameen, and A. A. Mohamed, "A new operational matrix based on jacobi wavelets for a class of variable-order fractional differential equations," Proceedings of the Romanian Academy Series A-Mathematics Physics Technical Sciences Information Science, vol. 18, no. 4, pp. 315-322, 2017.

[58] M. A. Zaky, S. S. Ezz-Eldien, E. H. Doha, J. A. Tenreiro Machado, and A. H. Bhrawy, "An efficient operational matrix technique for multidimensional variable-order time fractional 
diffusion equations," Journal of Computational and Nonlinear Dynamics, vol. 11, no. 6, 2016.

[59] M. A. Zaky, A. S. Hendy, A. A. Alikhanov, and V. G. Pimenov, "Numerical analysis of multi-term time-fractional nonlinear subdiffusion equations with time delay: what could possibly go wrong?," Communications in Nonlinear Science and Numerical Simulation, vol. 96, article 105672, 2021.

[60] A. H. Bhrawy and M. A. Zaky, "Numerical simulation for twodimensional variable-order fractional nonlinear cable equation," Nonlinear Dynamics, vol. 80, no. 1-2, pp. 101-116, 2015.

[61] A. H. Bhrawy, M. A. Zaky, and J. F. Alzaidy, "Two shifted Jacobi-Gauss collocation schemes for solving twodimensional variable-order fractional Rayleigh-Stokes problem," Advances Difference Equations, vol. 2016, no. 1, 2016.

[62] F. Polito and Ž. Tomovski, "Some properties of Prabhakartype fractional calculus operators," 2015, https://arxiv.org/ abs/1508.03224.

[63] A. A. Kilbas, M. Saigo, and R. K. Saxena, "Generalized MittagLeffler function and generalized fractional calculus operators," Integral Transforms and Special Functions, vol. 15, no. 1, pp. 31-49, 2004.

[64] K. Sun and M. Zhu, "Numerical algorithm to solve a class of variable order fractional integral-differential equation based on Chebyshev polynomials," Mathematical Problems in Engineering, vol. 2015, Article ID 902161, 10 pages, 2015.

[65] L. Hörmander and L. Hörmander, "The analysis of linear partial differential operators. III," Bulletin of the American Mathematical Society, vol. 16, pp. 161-167, 1987.

[66] P. Rahimkhani, Y. Ordokhani, and E. Babolian, "Fractionalorder Bernoulli wavelets and their applications," Applied Mathematical Modelling, vol. 40, no. 17-18, pp. 8087-8107, 2016.

[67] G. Doetsch, Introduction to the Theory and Application of the Laplace Transformation, Springer Science \& Business Media, 2012.

[68] R. Gorenflo, A. A. Kilbas, F. Mainardi, and S. V. Rogosin, Mittag-Leffler Functions, Related Topics and Applications, vol. 2, Springer, Berlin, 2014.

[69] M. H. Heydari, Z. Avazzadeh, Y. Yang, and C. Cattani, "A cardinal method to solve coupled nonlinear variable-order time fractional sine-Gordon equations," Computational and Applied Mathematics, vol. 39, no. 1, p. 2, 2020. 